\title{
Iron-catalyzed intra-intermolecular aminoazidation of alkenes
}

Sandra Abi Fayssal, Alessandro Giungi, Farouk Berhal*, Guillaume Prestat*

\section{Table of Contents}

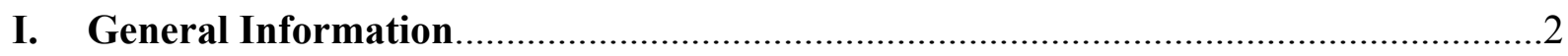

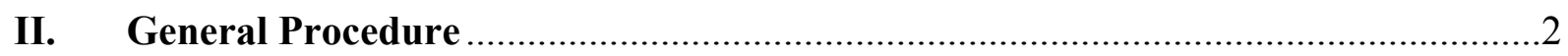

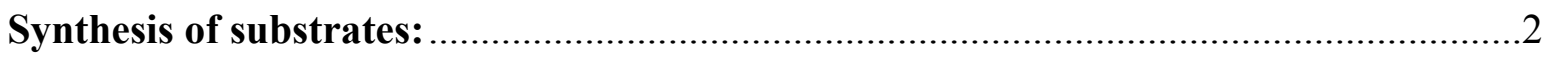

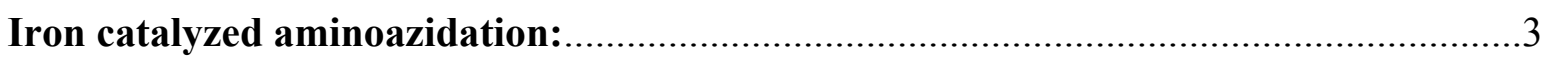

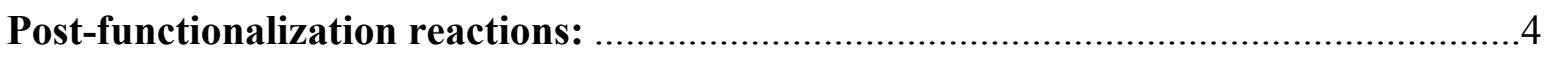

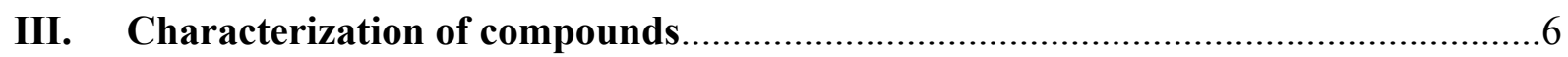

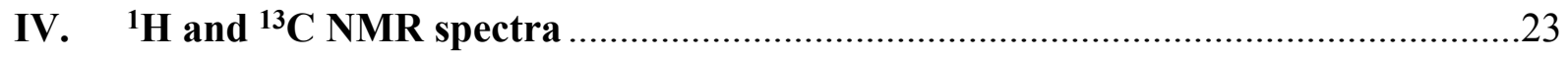

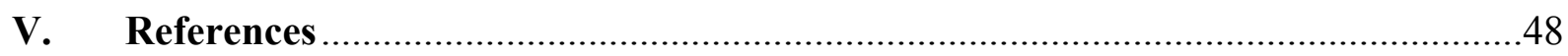




\section{General Information}

All reagents were purchased from chemical suppliers and used without further purification. Reactions were performed under an argon atmosphere. DCM was dried using a Pure Solv Micro solvent purification system. Dry THF and acetonitrile were purchased from chemical suppliers. Analytical thin layer chromatography was performed on commercial silica gel plates $60 \mathrm{~F}_{254}$. Flash column chromatography was performed on silica gel $60(40-63 \mu \mathrm{m})$. NMR spectra were recorded on a $500 \mathrm{MHz}$ spectrometer as specified. Chemical shifts $(\delta)$ are reported in ppm relative to tetramethylsilane $(\delta 0.00 \mathrm{ppm})$ or the $\mathrm{CHCl}_{3}$ residual peak $(\delta 7.26)$ or for the $\mathrm{CH}_{3} \mathrm{OH}$ residual peak $(\delta 3.31)$ for ${ }^{1} \mathrm{H}$ NMR. Chemical shifts of ${ }^{13} \mathrm{C}$ NMR are reported relative to $\mathrm{CDCl}_{3}(\delta 77.16)$ or $\mathrm{CD}_{3} \mathrm{OD}(\delta 49.00)$. Coupling constant $(J)$ are reported in Hertz unit $(\mathrm{Hz})$. Multiplicities are described with standard following abbreviations: $\mathrm{s}=$ singlet, $\mathrm{br}=$ broad, $\mathrm{d}=$ doublet, $\mathrm{t}=$ triplet, $\mathrm{q}=$ quadruplet, $\mathrm{m}=$ multiplet. Low resolution mass spectra (LRMS) were recorded with an ion trap mass analyzer under electrospray ionization (ESI) in positive or negative ionization mode detection or atmospheric pressure chemical ionization (APCI). High resolution mass spectra (HRMS) were recorded with a TOF mass analyzer under electrospray ionization (ESI) in positive or negative ionization mode detection, atmospheric pressure chemical ionization or atmospheric pressure photoionization (APPI). Melting points were measured on a Köfler bench. IR spectra were recorded on a FT-IR spectrophotometer, and the wavelengths reported in $\mathrm{cm}^{-1}$.

\section{General Procedure}

\section{Synthesis of substrates:}

Preparation of hydroxyl amine derivatives (scale: $0.500 \mathrm{~g}$ of starting material):

The allyl hydroxycarbamate derivatives were synthesized as below using a reported procedure. ${ }^{1}$

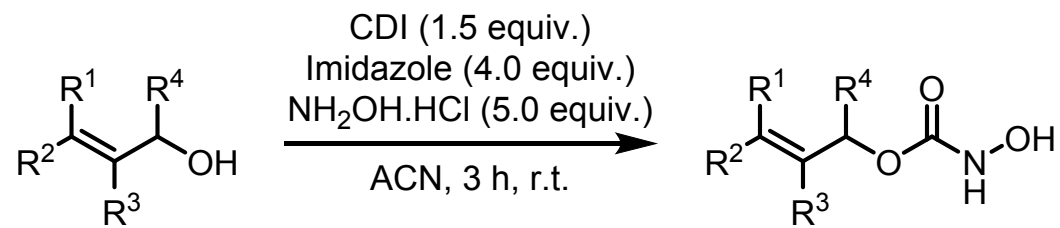

The carboxylic acids were commercially available or synthesized using a reported procedure. ${ }^{2}$ The hydroxypentenamide derivatives were prepared as below using a reported procedure. ${ }^{3}$

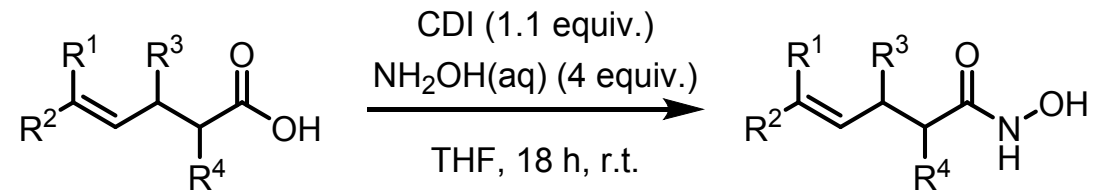


The allyl hydroxyl-methyl-urea derivatives were synthesized as below following our previous work. $^{4}$

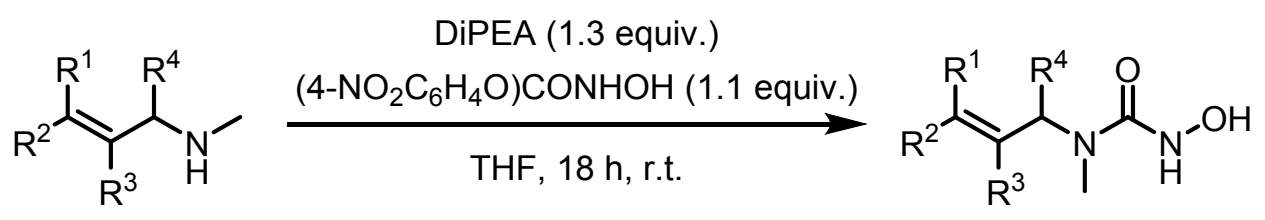

\section{Procedure A - acetylation of hydroxyl amine derivatives:}<smiles>[R]C([R])=C([R])C([R])[X]C(=O)NO</smiles>

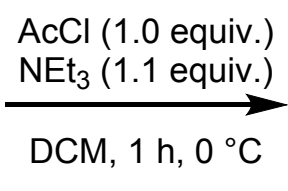<smiles>[R]C([R])=C([R])C([R])[X]C(=O)NOC(C)=O</smiles>
$\mathrm{X}=\mathrm{O}$, NMe or $\mathrm{CH}_{2}$

In a round-bottom flask, 1 equivalent of the desired allyl hydroxycarbamate derivative was placed in DCM $(\mathrm{C}=0.2 \mathrm{M})$ under argon at $0{ }^{\circ} \mathrm{C}$. Then 1.1 equiv. of triethylamine was added, followed by the dropwise addition of 1 equiv. of acetyl chloride. The solution was stirred at 0 ${ }^{\circ} \mathrm{C}$ for about $1 \mathrm{~h}$ (monitored by TLC). The reaction was quenched with a saturated aqueous solution of $\mathrm{NaHCO}_{3}$ and extracted with DCM. The combined organic phases were dried over $\mathrm{MgSO}_{4}$, filtered and concentrated under reduced pressure. The crude residue was then purified by silica gel chromatography using Pentane/AcOEt as eluent.

\section{Iron catalyzed aminoazidation:}

\section{Procedure B:}

In a sealed tube, $10 \mathrm{~mol} \mathrm{\%}$ of $\mathrm{Fe}(\mathrm{OAc})_{2}(5.1 \mathrm{mg}, 0.029 \mathrm{mmol})$ and $20 \mathrm{~mol} \%$ of phenanthroline $(10.5 \mathrm{mg}, 0.058 \mathrm{mmol})$ were placed in $\mathrm{ACN}(1.45 \mathrm{~mL}, \mathrm{C}=0.2 \mathrm{M})$ under argon. The solution was stirred for 10 minutes at room temperature and then 1.0 equiv. of the desired alkene substrate $(0.29 \mathrm{mmol})$ was added followed by 1.5 equiv. of $\mathrm{TMSN}_{3}(60 \mu \mathrm{L}$, $0.44 \mathrm{mmol}$ ). The deep red solution was stirred at $70{ }^{\circ} \mathrm{C}$ for $18 \mathrm{~h}$. The reaction mixture was then diluted with a saturated aqueous solution of $\mathrm{NaHCO}_{3}(10 \mathrm{~mL})$ to quench the unreacted $\mathrm{TMSN}_{3}$ reagent. The aqueous layer was extracted with DCM $(10 \mathrm{~mL}, 3$ times). The combined organic phases were dried over $\mathrm{MgSO}_{4}$, filtered and the solvents were removed under reduced pressure $\left(\mathrm{T}^{\circ} \mathrm{C}<50^{\circ} \mathrm{C}\right)$. The crude residue was purified by silica gel flash chromatography using cyclohexane / AcOEt as eluent. 


\section{Gram-scale experiments:}

\section{Product 4a}

In a round bottom flask, $2 \mathrm{~mol} \%$ of $\mathrm{Fe}(\mathrm{OAc})_{2}(22 \mathrm{mg}, 0.13 \mathrm{mmol})$ and $4 \mathrm{~mol} \%$ of phenanthroline (45 mg, $0.25 \mathrm{mmol}$ ) were placed in $\mathrm{ACN}(31.5 \mathrm{~mL}, \mathrm{C}=0.2 \mathrm{M})$ under argon. The solution was stirred for 10 minutes at room temperature and then 1.0 equiv. of the alkene substrate 3a was added $(1 \mathrm{~g}, 6.28 \mathrm{mmol})$ followed by 1.5 equiv. of $\mathrm{TMSN}_{3}(1.25 \mathrm{~mL}, 9.43$ mmol). The deep red solution was stirred at $70{ }^{\circ} \mathrm{C}$ for $18 \mathrm{~h}$. The reaction mixture was then concentrated at room temperature and diluted with a saturated aqueous solution of $\mathrm{NaHCO}_{3}$ $(20 \mathrm{~mL})$. The aqueous layer was extracted with DCM $(20 \mathrm{~mL}, 4$ times). The combined organic phases were dried over $\mathrm{MgSO}_{4}$, filtered and the solvent was removed under reduced pressure $\left(\mathrm{T}^{\circ} \mathrm{C}<50^{\circ} \mathrm{C}\right)$. The crude red residue was purified by silica gel flash chromatography using pentane / AcOEt as eluent and the pure product 4a was isolated as a white solid (710 mg, $80 \%$ Yield).

\section{Product 2b}

In a round bottom flask, $2 \mathrm{~mol} \%$ of $\mathrm{Fe}(\mathrm{OAc})_{2}(20 \mathrm{mg}, 0.12 \mathrm{mmol})$ and $4 \mathrm{~mol} \%$ of phenanthroline (42 mg, $0.23 \mathrm{mmol}$ ) were placed in ACN (29 mL, C = $0.2 \mathrm{M}$ ) under argon. The solution was stirred for 10 minutes at room temperature and then 1.0 equiv. of the alkene substrate $1 \mathbf{b}$ was added $(1 \mathrm{~g}, 5.81 \mathrm{mmol})$ followed by 1.5 equiv. of $\mathrm{TMSN}_{3}(1.15 \mathrm{~mL}, 8.71$ mmol). The deep red solution was stirred at $70{ }^{\circ} \mathrm{C}$ for $18 \mathrm{~h}$. The reaction mixture was then concentrated at room temperature and diluted with a saturated aqueous solution of $\mathrm{NaHCO}_{3}$ $(20 \mathrm{~mL})$. The aqueous layer was extracted with DCM (20 mL, 4 times). The combined organic phases were dried over $\mathrm{MgSO}_{4}$, filtered and the solvent was removed under reduced pressure $\left(\mathrm{T}^{\circ} \mathrm{C}<50^{\circ} \mathrm{C}\right)$. The crude red residue was purified by silica gel flash chromatography using pentane / AcOEt as eluent. The pure product $\mathbf{2 b}$ was isolated as a white solid $(570 \mathrm{mg}$, $63 \%$ Yield).

\section{Post-functionalization reactions:}

Procedure C: Boc-protection of the oxazolidinone moiety<smiles>N#CCC1COC(=O)N1</smiles>

4a

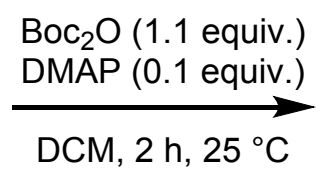

$\mathrm{DCM}, 2 \mathrm{~h}, 25^{\circ} \mathrm{C}$

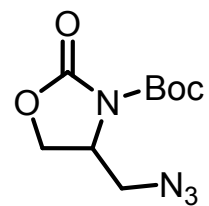

7 
In a round bottom flask 1 equivalent of compound $4 \mathbf{a}(100 \mathrm{mg}, 0.70 \mathrm{mmol})$ was placed in $\operatorname{DCM}(7 \mathrm{~mL}, 0.1 \mathrm{M})$ under argon. Then 1.1 equiv. of $\operatorname{Boc}_{2} \mathrm{O}(0.17 \mathrm{~mL}, 0.77 \mathrm{mmol})$ and 0.1 equiv. of DMAP ( $8.6 \mathrm{mg}, 0.070 \mathrm{mmol}$ ) were added. The solution was stirred at r.t. for $2 \mathrm{~h}$. The reaction mixture was concentrated under reduced pressure, then the crude residue was purified by silica gel flash chromatography using cyclohexane/ AcOEt (from 70/30 to 0/100) as eluent to obtain 7 as a white solid (157 $\mathrm{mg}, 92 \%)$.

\section{Procedure D: Cleavage of the oxazolidinone moiety}<smiles>NCC1COC(=O)N1C(=O)OCc1ccccc1</smiles>

7

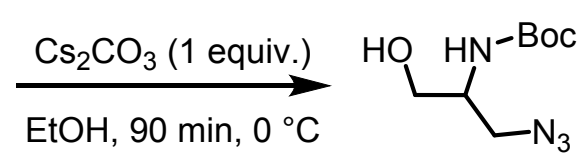

8

In a round bottom flask 1 equivalent of compound $7(137 \mathrm{mg}, 0.57 \mathrm{mmol})$ was placed in EtOH $(14 \mathrm{~mL}, 0.04 \mathrm{M})$ under argon. Then 1 equiv. of $\mathrm{Cs}_{2} \mathrm{CO}_{3}(184 \mathrm{mg}, 0.57 \mathrm{mmol})$ was added. The suspension was stirred at $0{ }^{\circ} \mathrm{C}$ for $1 \mathrm{~h} 30$. The reaction mixture was quenched with a saturated aqueous solution of $\mathrm{NH}_{4} \mathrm{Cl}(20 \mathrm{~mL})$ and extracted with $\mathrm{DCM}(20 \mathrm{~mL}, 4$ times $)$. The combined organic phases were dried over $\mathrm{MgSO}_{4}$, filtered and the solvents were removed under reduced pressure. The crude residue was purified by silica gel flash chromatography using cyclohexane/ AcOEt (from $80 / 20$ to 0/100 ratio) as eluent to obtain 8 as a colorless oil (103 mg, $84 \%)$.

\section{Procedure E: One-pot reduction of the azide moiety and Boc-protection}

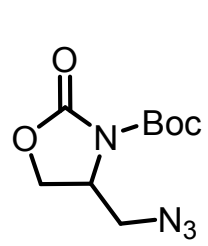

7

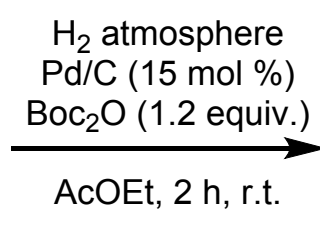

In a round bottom flask 1 equivalent of compound $7(170 \mathrm{mg}, 0.70 \mathrm{mmol})$ was placed in AcOEt $(5 \mathrm{~mL}, 0.14 \mathrm{M})$. Then 1.2 equiv. of $\mathrm{Boc}_{2} \mathrm{O}(0.18 \mathrm{~mL}, 0.84 \mathrm{mmol})$ and $15 \mathrm{~mol} \% \mathrm{w} / \mathrm{w}$ of $\mathrm{Pd}(10 \%$ on activated carbon, $26 \mathrm{mg}, 0.24 \mathrm{mmol})$ were added. The solution was stirred at r.t. for $2 \mathrm{~h}$ under $\mathrm{H}_{2}$ atmosphere (balloon). The reaction mixture was then filtered on celite ${ }^{\circledR}$ and the solid washed with ethyl acetate $(20 \mathrm{~mL})$. The solvent was removed under reduced pressure and the crude residue was purified by silica gel flash chromatography using 
pentane/AcOEt (from 80/20 to 50/50 ratio) as eluent to obtain 9 as a white solid (192 $\mathrm{mg}, 86$ $\%)$.

Procedure F: Azide-alkyne Huisgen cycloaddition<smiles>[14CH3]CC1COC(=O)N1</smiles>

1 equiv.

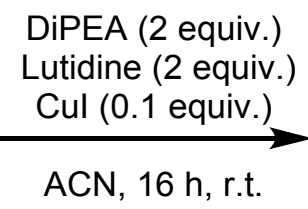

1 equiv.

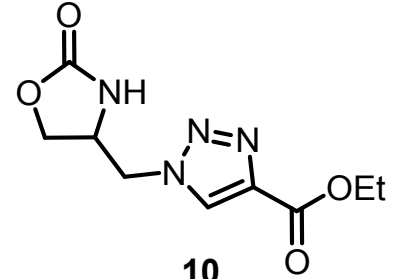

10

In a round bottom flask 1 equivalent of ethyl propiolate $(0.071 \mathrm{~mL}, 0.70 \mathrm{mmol})$ was placed in $\mathrm{ACN}(4.4 \mathrm{~mL}, 0.16 \mathrm{M})$ under argon. Then 2 equiv. of DiPEA (0.23 mL, $1.41 \mathrm{mmol}), 2$ equiv. of lutidine $(0.160 \mathrm{~mL}, 1.41 \mathrm{mmol})$ and 0.1 equiv. of $\mathrm{CuI}(0.013 \mathrm{mg}, 0.070 \mathrm{mmol})$ were sequentially added followed by 1 equiv. of the azido compound $4 \mathbf{a}(0.100 \mathrm{~g}, 0.70 \mathrm{mmol})$. The clear orange solution was stirred at r.t. for $16 \mathrm{~h}$. The solvent was then removed under reduced pressure and the residue solubilized with AcOEt $(10 \mathrm{~mL})$. The reaction mixture was quenched with a saturated aqueous solution of $\mathrm{NaCl}(10 \mathrm{~mL})$ and the aqueous phase extracted with AcOEt (10 mL, 10 times). The combined organic phases were dried over $\mathrm{MgSO}_{4}$, filtered and the solvent was removed under reduced pressure. The crude residue was purified by silica gel flash chromatography using AcOEt as eluent to obtain $\mathbf{1 0}$ as a pink white solid (140 mg, 83 $\%)$.

\section{Characterization of compounds}

\section{1b - 3-acetoxy-1-allyl-1-methylurea}<smiles>C=CCN(C)C(=O)NOC(C)=O</smiles>

White solid; $231 \mathrm{mg}, 87 \%$ Yield (Procedure A using the corresponding substrate $200 \mathrm{mg}$ ); m.p. $66{ }^{\circ} \mathrm{C} ;{ }^{1} \mathrm{H} \mathrm{RMN}\left(\mathrm{CDCl}_{3}, 500 \mathrm{MHz}, \mathrm{ppm}\right) \delta=8.23$ (brs, $\left.1 \mathrm{H}\right), 5.79$ (ddt, $J=5.5 \mathrm{~Hz}, 10.5$ Hz, 16.5 Hz, 1H), 5.27-5.21 (m, 2H), 3.90 (dt, $J=1.5 \mathrm{~Hz}, 5 \mathrm{~Hz}, 2 \mathrm{H}), 2.94$ (s, 3H), 2.21 (s, $3 \mathrm{H}) ;{ }^{13} \mathrm{C} \mathrm{RMN}\left(\mathrm{CDCl}_{3}, 125 \mathrm{MHz}, \mathrm{ppm}\right) \delta=170.9,157.8,132.3,117.6,51.3,34.2,18.6$; IR ( $v$ $\left.=\mathrm{cm}^{-1}\right): 3219,2983,2933,1784,1670,1493,1195$. 


\section{1c - 3-acetoxy-1-cinnamyl-1-methylurea}

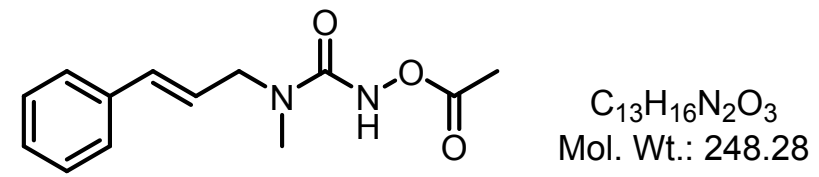

Colourless oil; $244 \mathrm{mg}, 60 \%$ Yield (Procedure A using the corresponding substrate $340 \mathrm{mg}$ ); ${ }^{1} \mathrm{H} \mathrm{RMN}\left(\mathrm{CDCl}_{3}, 500 \mathrm{MHz}, \mathrm{ppm}\right) \delta=8.28$ (brs, $\left.1 \mathrm{H}\right), 7.40-7.37(\mathrm{~m}, 2 \mathrm{H}), 7.34-7.30(\mathrm{~m}, 2 \mathrm{H})$, 7.27-7.25 (m, 1H), $6.56(\mathrm{~d}, J=16 \mathrm{~Hz}, 1 \mathrm{H}), 6.15(\mathrm{dt}, J=6 \mathrm{~Hz}, 16 \mathrm{~Hz}, 1 \mathrm{H}), 4.07$ (dd, $J=1.5$ $\mathrm{Hz}, 6 \mathrm{~Hz}, 2 \mathrm{H}), 2.99$ (s, 3H), $2.23(\mathrm{~s}, 3 \mathrm{H}) ;{ }^{13} \mathrm{C} \mathrm{RMN}\left(\mathrm{CDCl}_{3}, 125 \mathrm{MHz}, \mathrm{ppm}\right) \delta=170.9,157.9$, 136.3, 132.9, 128.8, 128.1, 126.6, 123.5, 50.9, 34.2, 18.6; IR $\left(v=\mathrm{cm}^{-1}\right): 3209,3022,2968$, 2938, 1784, 1670, 1496, 1192, 748.

\section{1d - 3-acetoxy-1-(cyclohex-2-en-1-yl)-1-methylurea}<smiles>CC(=O)ONC(=O)N(C)C1C=CCCC1</smiles>

White solid; $133 \mathrm{mg}, 82 \%$ Yield; (Procedure A using the corresponding substrate $130 \mathrm{mg}$ ); m.p. 84-85 ${ }^{\circ} \mathrm{C}$; ${ }^{1} \mathrm{H} \mathrm{RMN}\left(\mathrm{CDCl}_{3}, 500 \mathrm{MHz}, \mathrm{ppm}\right) \delta=8.25$ (brs, $\left.1 \mathrm{H}\right), 5.98-5.93$ (m, 1H), 5.49$5.44(\mathrm{~m}, 1 \mathrm{H}), 4.72(\mathrm{brs}, 1 \mathrm{H}), 2.81(\mathrm{~s}, 3 \mathrm{H}), 2.23(\mathrm{~s}, 3 \mathrm{H}), 2.04-1.99(\mathrm{~m}, 2 \mathrm{H}), 1.91-1.85(\mathrm{~m}, 1 \mathrm{H})$,

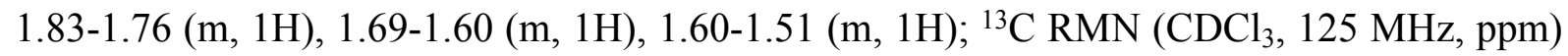
$\delta=171.0,157.7,132.6,127.5,52.8,29.3,26.7,24.6,21.3,18.7$; IR $\left(v=\mathrm{cm}^{-1}\right): 3208,3023$, 2936, 2858, 2834, 1785, 1664, 1482, 1193, 1152, 1060, 743.

\section{1e - 3-acetoxy-1-methyl-1-(3-methylbut-2-en-1-yl)urea}<smiles>CC(=O)ONC(=O)N(C)CC=C(C)C</smiles>

Colourless oil; $74 \mathrm{mg}, 58 \%$ Yield (Procedure A using the corresponding substrate $100 \mathrm{mg}$ ); ${ }^{1} \mathrm{H} \mathrm{RMN}\left(\mathrm{CDCl}_{3}, 500 \mathrm{MHz}, \mathrm{ppm}\right) \delta=8.28$ (brs, $\left.1 \mathrm{H}\right), 5.21-5.15(\mathrm{~m}, 1 \mathrm{H}), 3.87(\mathrm{~d}, J=7 \mathrm{~Hz}$, 2H), 2.91 (s, 3H), 2.21 (s, 3H), 1.75 (s, 3H), 1.70 (s, 3H); $\left.{ }^{13} \mathrm{C} \mathrm{RMN} \mathrm{(CDCl} 3,125 \mathrm{MHz}, \mathrm{ppm}\right)$ $\delta=170.8,157.7,137.5,118.9,46.6,34.0,25.8,18.7,18.0 ;$ IR $\left(v=\mathrm{cm}^{-1}\right): 3208,2971,2931$, $1785,1665,1490,1189$.

\section{1f - 3-acetoxy-1-methyl-1-(2-methylallyl)urea}




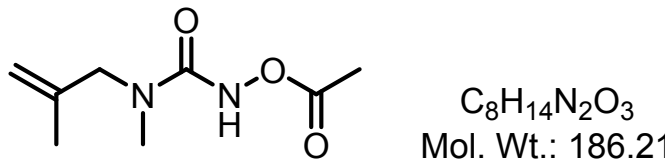

Colourless oil; $144 \mathrm{mg}, 77 \%$ Yield (Procedure A using the corresponding substrate $144 \mathrm{mg}$ ); ${ }^{1} \mathrm{H} \mathrm{RMN}\left(\mathrm{CDCl}_{3}, 500 \mathrm{MHz}, \mathrm{ppm}\right) \delta=8.20$ (brs, 1H), 4.96 (s, 1H), 4.89 (s, 1H), 3.82 (s, 2H), 2.94 (s, 3H), 2.21 (s, 3H), $1.72(\mathrm{~s}, 3 \mathrm{H}) ;{ }^{13} \mathrm{C} \mathrm{RMN}\left(\mathrm{CDCl}_{3}, 125 \mathrm{MHz}, \mathrm{ppm}\right) \delta=170.9,158.0$, 140.2, 112.4, 54.7, 34.6, 19.8, 18.6; IR $\left(v=\mathrm{cm}^{-1}\right): 3220,2978,2940,1785,1670,1493,1192$, 898.

3a - allyl acetoxycarbamate

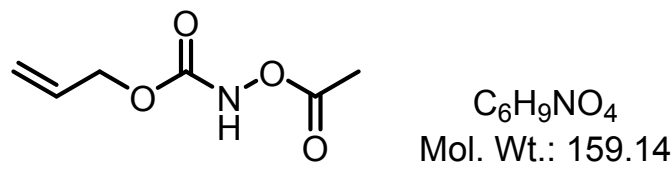

Colourless oil; $1.8 \mathrm{~g}, 74 \%$ Yield (Procedure A using the corresponding substrate $1.8 \mathrm{~g}$ ); ${ }^{1} \mathrm{H}$ $\mathrm{RMN}\left(\mathrm{CDCl}_{3}, 500 \mathrm{MHz}, \mathrm{ppm}\right) \delta=8.13$ (brs, 1H), 5.97-5.87 (m, 1H), 5.35 (dd, $J=1.5 \mathrm{~Hz}, 17$ $\mathrm{Hz}, 1 \mathrm{H}), 5.27(\mathrm{dd}, J=1.5 \mathrm{~Hz}, 10.5 \mathrm{~Hz}, 1 \mathrm{H}), 4.68(\mathrm{dt}, J=15 \mathrm{~Hz}, 6 \mathrm{~Hz}, 2 \mathrm{H}), 2.21(\mathrm{~s}, 3 \mathrm{H}) ;{ }^{13} \mathrm{C}$ $\mathrm{RMN}\left(\mathrm{CDCl}_{3}, 125 \mathrm{MHz}, \mathrm{ppm}\right) \delta=169.9,156.3,131.5,119.2,67.3,18.4 ; \mathrm{IR}\left(v=\mathrm{cm}^{-1}\right)$ : 3264, $3085,2956,1793,1737,1256,1182,1103$.

\section{3b - cinnamyl acetoxycarbamate}

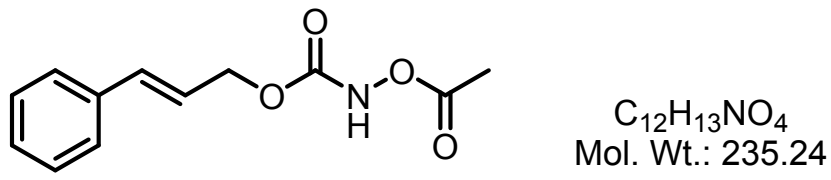

White solid; $674 \mathrm{mg}, 97 \%$ Yield (Procedure A using the corresponding substrate $572 \mathrm{mg}$ ); m.p. 69-71 ${ }^{\circ} \mathrm{C} ;{ }^{1} \mathrm{H} \mathrm{RMN}\left(\mathrm{CDCl}_{3}, 500 \mathrm{MHz}, \mathrm{ppm}\right) \delta=8.19$ (brs, $\left.1 \mathrm{H}\right), 7.41-7.37$ (m, 2H), 7.35$7.30(\mathrm{~m}, 2 \mathrm{H}), 7.29-7.25(\mathrm{~m}, 1 \mathrm{H}), 6.68(\mathrm{~d}, J=15.5 \mathrm{~Hz}, 1 \mathrm{H}), 6.27(\mathrm{dt}, J=6 \mathrm{~Hz}, 15.5 \mathrm{~Hz}, 1 \mathrm{H})$, $4.84(\mathrm{dd}, J=1 \mathrm{~Hz}, 6.5 \mathrm{~Hz}, 2 \mathrm{H}), 2.22(\mathrm{~s}, 3 \mathrm{H}) ;{ }^{13} \mathrm{C} \mathrm{RMN}\left(\mathrm{CDCl}_{3}, 125 \mathrm{MHz}, \mathrm{ppm}\right) \delta=169.9$, $156.4,136.1,135.2,128.8,128.4,126.9,122.3,67.4,18.4$; IR $\left(v=\mathrm{cm}^{-1}\right): 3201,2970,1789$, 1716, 1496, 1373, 1271, 1194, 1109, 974, 864, 745.

3c - (E)-3-(4-chlorophenyl)allyl acetoxycarbamate<smiles>CC(=O)ONC(=O)OC/C=C/c1ccc(Cl)cc1</smiles> 
Pale yellow solid; $217 \mathrm{mg}$, 69 \% Yield (Procedure A using the corresponding substrate 265 mg); m.p. 99-101 ${ }^{\circ} \mathrm{C}$; ${ }^{1} \mathrm{H}$ RMN $\left(\mathrm{CDCl}_{3}, 500 \mathrm{MHz}, \mathrm{ppm}\right) \delta=8.14$ (brs, $\left.1 \mathrm{H}\right), 7.33-7.27$ (m, 4H), $6.63(\mathrm{~d}, J=16 \mathrm{~Hz}, 1 \mathrm{H}), 6.24(\mathrm{dt}, J=6.5 \mathrm{~Hz}, 16 \mathrm{~Hz}, 1 \mathrm{H}), 4.83(\mathrm{~d}, J=6.5 \mathrm{~Hz}, 2 \mathrm{H}), 2.22$ (s, 3H); ${ }^{13} \mathrm{C} \mathrm{RMN}\left(\mathrm{CDCl}_{3}, 125 \mathrm{MHz}, \mathrm{ppm}\right) \delta=169.9,156.3,134.6,134.1,133.8,129.0$, 128.1, 123.0, 67.1, 18.4; IR $\left(v=\mathrm{cm}^{-1}\right): 3675,3205,2988,2972,2901,1797,1726,1491$, $1269,1182,1090,1075,1066,1057,857$.

3d - (E)-3-(4-nitrophenyl)allyl acetoxycarbamate

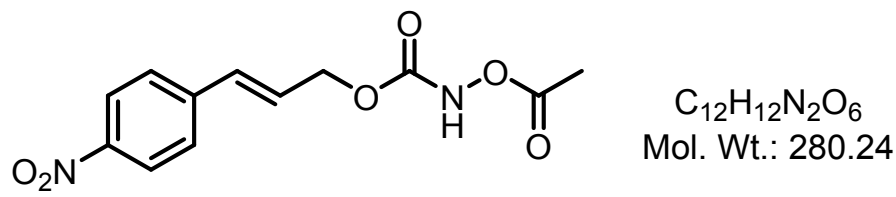

Yellow solid; $158 \mathrm{mg}, 58 \%$ Yield (Procedure A using the corresponding substrate $230 \mathrm{mg}$ );

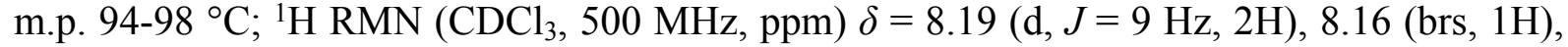
$7.52(\mathrm{~d}, J=9 \mathrm{~Hz}, 2 \mathrm{H}), 6.73(\mathrm{~d}, J=16 \mathrm{~Hz}, 1 \mathrm{H}), 6.44(\mathrm{dt}, J=6 \mathrm{~Hz}, 16 \mathrm{~Hz}, 1 \mathrm{H}), 4.89(\mathrm{dd}, J=1$ $\mathrm{Hz}, 5.5 \mathrm{~Hz}, 2 \mathrm{H}), 2.23(\mathrm{~s}, 3 \mathrm{H}) ;{ }^{13} \mathrm{C} \mathrm{RMN}\left(\mathrm{CDCl}_{3}, 125 \mathrm{MHz}, \mathrm{ppm}\right) \delta=169.9,156.1,147.5$, 142.5, 132.1, 127.4, 127.3, 124.2, 66.4, 18.4; IR $\left(v=\mathrm{cm}^{-1}\right): 3198,2968,2925,1784,1724$, $1517,1341,1262,1181,1110,977,971,863,770,743$.

3e - (E)-3-(2-methoxyphenyl)allyl acetoxycarbamate

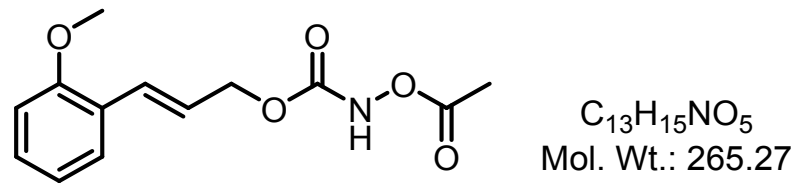

Colourless oil; $404 \mathrm{mg}, 63$ \% Yield (Procedure A using the corresponding substrate $540 \mathrm{mg}$ ); ${ }^{1} \mathrm{H} \mathrm{RMN}\left(\mathrm{CDCl}_{3}, 500 \mathrm{MHz}, \mathrm{ppm}\right) \delta=8.14$ (brs, $\left.1 \mathrm{H}\right), 7.43$ (d, $\left.J=8 \mathrm{~Hz}, 1 \mathrm{H}\right), 7.28-7.22$ (m, $1 \mathrm{H}), 7.00(\mathrm{~d}, J=16 \mathrm{~Hz}, 1 \mathrm{H}), 6.93(\mathrm{t}, J=7.5 \mathrm{~Hz}, 1 \mathrm{H}), 6.87(\mathrm{~d}, J=8.5 \mathrm{~Hz}, 1 \mathrm{H}), 6.31$ (dt, $J=7$ $\mathrm{Hz}, 16 \mathrm{~Hz}, 1 \mathrm{H}), 4.85(\mathrm{~d}, J=7 \mathrm{~Hz}, 2 \mathrm{H}), 3.85(\mathrm{~s}, 3 \mathrm{H}), 2.22(\mathrm{~s}, 3 \mathrm{H}) ;{ }^{13} \mathrm{C} \mathrm{RMN}\left(\mathrm{CDCl}_{3}, 125\right.$ MHz, ppm) $\delta=169.9,157.1,156.4,130.4,129.5,127.5,125.1,122.9,120.8,111.0,68.0$, 55.6, 18.5; IR $\left(v=\mathrm{cm}^{-1}\right): 3267,2942,2839,1793,1752,1736,1490,1464,1245,1182,1101$, 1027, 976, 753.

\section{3f - (E)-3-(4-methoxyphenyl)allyl acetoxycarbamate}




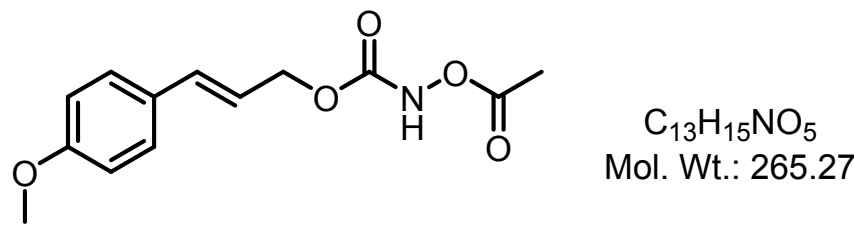

Pale yellow solid; $262 \mathrm{mg}$, $62 \%$ Yield (Procedure A using the corresponding substrate 360 mg); m.p. 64-68 ${ }^{\circ} \mathrm{C} ;{ }^{1} \mathrm{H} \mathrm{RMN}\left(\mathrm{CDCl}_{3}, 500 \mathrm{MHz}, \mathrm{ppm}\right) \delta=8.18(\mathrm{~s}, 1 \mathrm{H}), 7.32(\mathrm{~d}, J=8.5 \mathrm{~Hz}$, $2 \mathrm{H}), 6.86(\mathrm{~d}, J=8.5 \mathrm{~Hz}, 2 \mathrm{H}), 6.62(\mathrm{~d}, J=15.5 \mathrm{~Hz}, 1 \mathrm{H}), 6.14(\mathrm{dt}, J=6.5 \mathrm{~Hz}, 16 \mathrm{~Hz}, 1 \mathrm{H}), 4.81$ $(\mathrm{dd}, J=1.5 \mathrm{~Hz}, 6.5 \mathrm{~Hz}, 2 \mathrm{H}), 3.81(\mathrm{~s}, 3 \mathrm{H}), 2.21(\mathrm{~s}, 3 \mathrm{H}) ;{ }^{13} \mathrm{C} \mathrm{RMN}\left(\mathrm{CDCl}_{3}, 125 \mathrm{MHz}, \mathrm{ppm}\right) \delta$ $=169.9,159.9,156.4,135.1,128.8,128.1,119.9,114.2,67.7,55.4,18.4 ; \mathrm{IR}\left(v=\mathrm{cm}^{-1}\right): 3169$, 2965, 2841, 1798, 1709, 1607, 1512, 1269, 1253, 1183, 1174, 1099, 1028, 966, 843, 805, 766.

\section{3g - 2-methylallyl acetoxycarbamate}

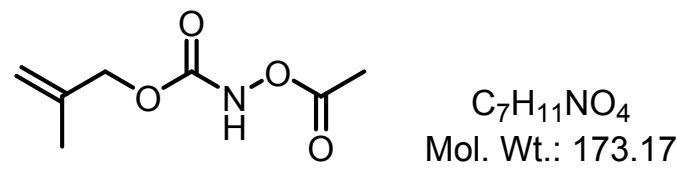

Colourless oil; $577 \mathrm{mg}, 62 \%$ Yield (Procedure A using the corresponding substrate $700 \mathrm{mg}$ ); ${ }^{1} \mathrm{H} \mathrm{RMN}\left(\mathrm{CDCl}_{3}, 500 \mathrm{MHz}, \mathrm{ppm}\right) \delta=8.16$ (brs, $\left.1 \mathrm{H}\right), 5.01-4.99(\mathrm{~m}, 1 \mathrm{H}), 4.97-4.94(\mathrm{~m}, 1 \mathrm{H})$, 4.60 (brs, 2H), 2.21 (s, 3H), 1.76 (s, 3H); ${ }^{13} \mathrm{C} \mathrm{RMN}\left(\mathrm{CDCl}_{3}, 125 \mathrm{MHz}, \mathrm{ppm}\right) \delta=169.9,156.4$, 139.3, 113.8, 69.9, 19.4, 18.4; IR $\left(v=\mathrm{cm}^{-1}\right): 3266,2976,1794,1738,1453,1263,1182,1105$, $856,768$.

\section{3h - (E)-2-methyl-3-phenylallyl acetoxycarbamate}

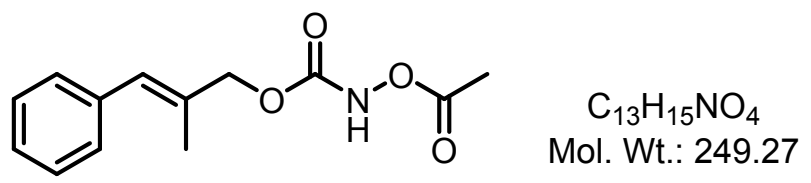

White solid; $418 \mathrm{mg}, 58 \%$ Yield; (Procedure A using the corresponding substrate $604 \mathrm{mg}$ ); m.p. 74-75 ${ }^{\circ} \mathrm{C} ;{ }^{1} \mathrm{H} \mathrm{RMN}\left(\mathrm{CDCl}_{3}, 500 \mathrm{MHz}, \mathrm{ppm}\right) \delta=8.19$ (brs, $\left.1 \mathrm{H}\right), 7.36-7.31$ (m, 2H), 7.29$7.23(\mathrm{~m}, 3 \mathrm{H}), 6.55$ (s, 1H), 4.75 (brs, 2H), 2.21 (s, 3H), 1.90 (d, $J=1.5 \mathrm{~Hz}, 3 \mathrm{H}) ;{ }^{13} \mathrm{C} \mathrm{RMN}$ $\left(\mathrm{CDCl}_{3}, 125 \mathrm{MHz}, \mathrm{ppm}\right) \delta=169.9,156.5,136.9,132.1,129.3,129.1,128.3,127.1,72,4$, 18.4, 15.5; IR $\left(v=\mathrm{cm}^{-1}\right): 3173,2992,1804,1710,1513,1297,1274,1182,1125,853,704$.

\section{$3 i$ - cyclohex-2-en-1-yl acetoxycarbamate}


$\nabla_{\mathrm{O}_{\mathrm{H}}} \mathrm{ll}_{\mathrm{O}}^{\mathrm{O}}$

$\mathrm{C}_{9} \mathrm{H}_{13} \mathrm{NO}_{4}$

Mol. Wt.: 199.21

Colourless oil; $291 \mathrm{mg}, 42 \%$ Yield; (Procedure A, using the corresponding substrate 550 $\mathrm{mg}) ;{ }^{1} \mathrm{H} \mathrm{RMN}\left(\mathrm{CDCl}_{3}, 500 \mathrm{MHz}, \mathrm{ppm}\right) \delta=8.07$ (brs, $\left.1 \mathrm{H}\right), 6.02-5.95$ (m, 1H), 5.77-5.70 (m, $1 \mathrm{H}), 5.30-5.22(\mathrm{~m}, 1 \mathrm{H}), 2.20(\mathrm{~s}, 3 \mathrm{H}), 2.13-2.04(\mathrm{~m}, 1 \mathrm{H}), 2.03-1.94(\mathrm{~m}, 1 \mathrm{H}), 1.91-1.85(\mathrm{~m}$, $1 \mathrm{H}), 1.84-1.77(\mathrm{~m}, 1 \mathrm{H}), 1.76-1.68(\mathrm{~m}, 1 \mathrm{H}), 1.67-1.60(\mathrm{~m}, 1 \mathrm{H}) ;{ }^{13} \mathrm{C} \mathrm{RMN}\left(\mathrm{CDCl}_{3}, 125 \mathrm{MHz}\right.$, ppm) $\delta=170,0156.4,133.7,125.0,71.1,28.4,24.9,18.7,18.5 ;$ IR $\left(v=\mathrm{cm}^{-1}\right): 3175,2988$, 2939, 1799, 1710, 1272, 1183, 1125, 853, 704.

\section{3j - 3-methylbut-2-en-1-yl acetoxycarbamate}

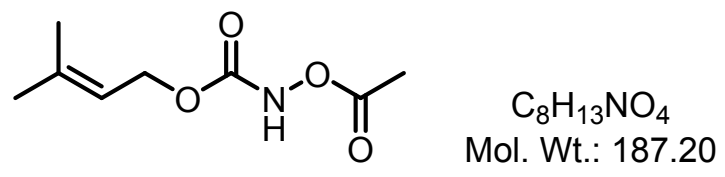

Pale yellow oil; $421 \mathrm{mg}, 54 \%$ Yield (Procedure A using the corresponding substrate $600 \mathrm{mg}$ ); ${ }^{1} \mathrm{H} \mathrm{RMN}\left(\mathrm{CDCl}_{3}, 500 \mathrm{MHz}, \mathrm{ppm}\right) \delta=8.10(\mathrm{~s}, 1 \mathrm{H}), 5.38-5.32(\mathrm{~m}, 1 \mathrm{H}), 4.68(\mathrm{~d}, J=7.5 \mathrm{~Hz}$, 2H), 2.20 (s, 3H), 1.76 (s, 3H), $1.72(\mathrm{~s}, 3 \mathrm{H}) ;{ }^{13} \mathrm{C} \mathrm{RMN}\left(\mathrm{CDCl}_{3}, 125 \mathrm{MHz}, \mathrm{ppm}\right) \delta=169.9$, 156.7, 140.6, 117.9, 63.7, 25.9, 18.5, 18.2; IR $\left(v=\mathrm{cm}^{-1}\right): 3258,3166,2971,1796,1738,1709$, $1512,1253,1183,1098,1028,966,843,767$.

\section{5 a - $N$-acetoxypent-4-enamide}

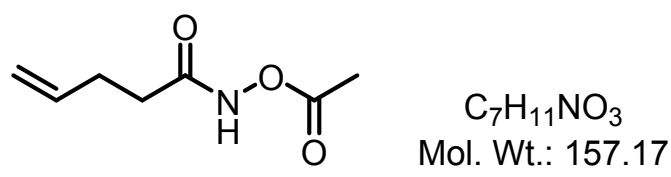

White solid; $379 \mathrm{mg}, 60 \%$ Yield (Procedure A using the corresponding substrate $466 \mathrm{mg}$ ); m.p. 55-62 ${ }^{\circ} \mathrm{C} ;{ }^{1} \mathrm{H} \mathrm{RMN}\left(\mathrm{CDCl}_{3}, 500 \mathrm{MHz}, \mathrm{ppm}\right) \delta=8.95$ (brs, $\left.1 \mathrm{H}\right), 5.83$ (ddt, $J=6.5 \mathrm{~Hz}, 10$ $\mathrm{Hz}, 17 \mathrm{~Hz}, 1 \mathrm{H}), 5.10$ (dd, $J=1.5 \mathrm{~Hz}, 17 \mathrm{~Hz}, 1 \mathrm{H}), 5.05$ (dd, $J=1 \mathrm{~Hz}, 10 \mathrm{~Hz}, 1 \mathrm{H}), 2.496-2.40$ (m, 2H), 2.39-2.33 (m, 2H), $2.22(\mathrm{~s}, 3 \mathrm{H}) ;{ }^{13} \mathrm{C} \mathrm{RMN}\left(\mathrm{CDCl}_{3}, 125 \mathrm{MHz}, \mathrm{ppm}\right) \delta=174.2,169.0$, 136.4, 116.4, 32.5, 28.9, 18.4; IR $\left(v=\mathrm{cm}^{-1}\right): 3147,2978,2901,1793,1656,1180,852$.

$5 b-(E)-N$-acetoxy-5-phenylpent-4-enamide<smiles>CC(=O)ONC(=O)CC/C=C/c1ccccc1</smiles> 
White solid; $512 \mathrm{mg}, 76 \%$ Yield (Procedure A using the corresponding substrate $552 \mathrm{mg}$ ); m.p. 102-104 ${ }^{\circ} \mathrm{C} ;{ }^{1} \mathrm{H} \mathrm{RMN}\left(\mathrm{CDCl}_{3}, 500 \mathrm{MHz}, \mathrm{ppm}\right) \delta=8.91$ (brs, $\left.1 \mathrm{H}\right), 7.36-7.32(\mathrm{~m}, 2 \mathrm{H})$, 7.31-7.26 (m, 2H), 7.24-7.18 (m, 1H), 6.47 (d, $J=16 \mathrm{~Hz}, 1 \mathrm{H}), 6.26-6.17(\mathrm{~m}, 1 \mathrm{H}), 2.60$ (q, $J=$ $7 \mathrm{~Hz}, 2 \mathrm{H}), 2.43(\mathrm{t}, J=7.5 \mathrm{~Hz}, 2 \mathrm{H}), 2.22(\mathrm{~s}, 3 \mathrm{H}) ;{ }^{13} \mathrm{C} \mathrm{RMN}\left(\mathrm{CDCl}_{3}, 125 \mathrm{MHz}, \mathrm{ppm}\right) \delta=$ 170.6, 168.9, 137.3, 131.7, 128.7, 127.9, 127.4, 126.3, 32.9, 28.4, 18.4; IR $\left(v=\mathrm{cm}^{-1}\right): 3160$, 2978, 1800, 1794, 1658, 1522, 1444, 1370, 1180, 1052, 973, 851, 743.

\section{5d - $N$-acetoxyhex-4-enamide (cis + trans)}

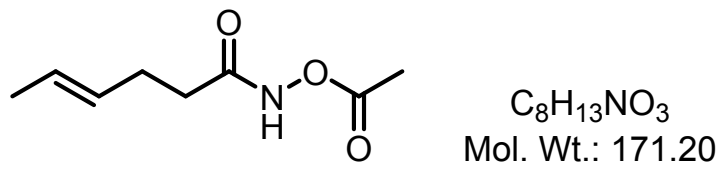

White solid; $484 \mathrm{mg}, 83 \%$ Yield; (Procedure A using the corresponding substrate $440 \mathrm{mg}$ ); m.p. 80-82 ${ }^{\circ} \mathrm{C}$; ${ }^{1} \mathrm{H} \mathrm{RMN}\left(\mathrm{CDCl}_{3}, 500 \mathrm{MHz}, \mathrm{ppm}\right) \delta=5.58-5.49(\mathrm{~m}, 1 \mathrm{H}), 5.47-5.38(\mathrm{~m}, 1 \mathrm{H})$, 2.39-2.33 (m, 2H), 2.33-2.28 (m, 2H), $2.22(\mathrm{~s}, 3 \mathrm{H}), 1.65$ (dd, $J=1 \mathrm{~Hz}, 6.5 \mathrm{~Hz}, 3 \mathrm{H}) ;{ }^{13} \mathrm{C} \mathrm{RMN}$ $\left(\mathrm{CDCl}_{3}, 125 \mathrm{MHz}, \mathrm{ppm}\right) \delta=170.8,169.0,128.9,127.3,33.3,28.0,18.5,18.0 ; \mathrm{IR}\left(\mathrm{v}=\mathrm{cm}^{-1}\right)$ : $3143,2963,1798,1792,1655,1179,1050,965,847$.

\section{5e - $N$-acetoxy-4-methylpent-4-enamide}

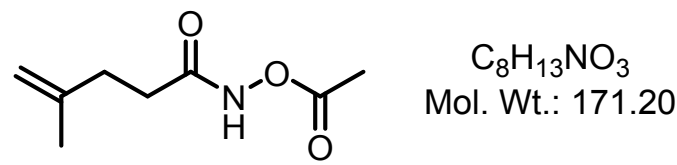

White solid; $179 \mathrm{mg}, 84 \%$ Yield; (Procedure A using the corresponding substrate $160 \mathrm{mg}$ ); m.p. $85{ }^{\circ} \mathrm{C}$; ${ }^{1} \mathrm{H} \mathrm{RMN}\left(\mathrm{CDCl}_{3}, 500 \mathrm{MHz}, \mathrm{ppm}\right) \delta=9.00$ (brs, $\left.1 \mathrm{H}\right), 4.79$ (s, 1H), $4.74(\mathrm{~s}, 1 \mathrm{H})$, 2.45-2.35 (m, 4H), 2.22 (s, 3H), $1.75(\mathrm{~s}, 3 \mathrm{H}) ;{ }^{13} \mathrm{C} \mathrm{RMN}\left(\mathrm{CDCl}_{3}, 125 \mathrm{MHz}, \mathrm{ppm}\right) \delta=171.0$, 169.0, 143.9, 111.2, 32.6, 31.5, 22.5, 18.4; IR $\left(v=\mathrm{cm}^{-1}\right): 3143,2968,2905,1795,1654,1179$, 1025,879 .

\section{5 - $N$-acetoxy-3-methylpent-4-enamide}

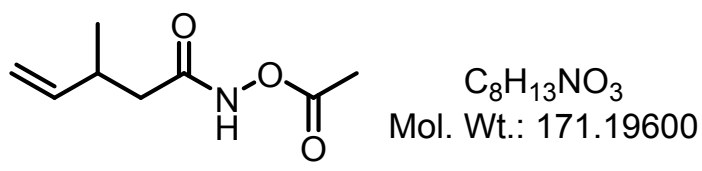

White solid; $185 \mathrm{mg}, 80 \%$ Yield; (Procedure A using the corresponding substrate $174 \mathrm{mg}$ ); m.p. 86-88 ${ }^{\circ} \mathrm{C} ;{ }^{1} \mathrm{H} \mathrm{RMN}\left(\mathrm{CDCl}_{3}, 500 \mathrm{MHz}, \mathrm{ppm}\right) \delta=8.92$ (brs, $\left.1 \mathrm{H}\right), 5.79$ (ddd, $J=7 \mathrm{~Hz}$, $10.5 \mathrm{~Hz}, 17 \mathrm{~Hz}, 1 \mathrm{H}), 5.08$ (dt, $J=1.5 \mathrm{~Hz}, 17 \mathrm{~Hz}, 1 \mathrm{H}), 5.02$ (dt, $J=1 \mathrm{~Hz}, 10.5 \mathrm{~Hz}, 1 \mathrm{H}), 2.80-$ 
$2.69(\mathrm{~m}, 1 \mathrm{H}), 2.30(\mathrm{dd}, J=7 \mathrm{~Hz}, 14.5 \mathrm{~Hz}, 1 \mathrm{H}), 2.22$ (s, 3H), 2.20 (dd, $J=7 \mathrm{~Hz}, 14 \mathrm{~Hz}, 1 \mathrm{H})$, $1.09(\mathrm{~d}, J=6.5 \mathrm{~Hz}, 3 \mathrm{H}) ;{ }^{13} \mathrm{C} \mathrm{RMN}\left(\mathrm{CDCl}_{3}, 125 \mathrm{MHz}, \mathrm{ppm}\right) \delta=170.0,168.9,142.2,114.3$, 40.2, 34.4, 19.7, 18.4; IR $\left(v=\mathrm{cm}^{-1}\right): 3161,2977,2928,2868,1794,1661,1186,852$.

2b - 4-(azidomethyl)-1-methylimidazolidin-2-one<smiles>CN1CC(CN)NC1=O</smiles>

\section{$\mathrm{C}_{5} \mathrm{H}_{9} \mathrm{~N}_{5} \mathrm{O}$}

Mol. Wt.: 155.16

Beige solid; $32 \mathrm{mg}, 71 \%$ Yield (Procedure B using substrate 1b $50 \mathrm{mg}$ ); m.p. $72-74{ }^{\circ} \mathrm{C}$; ${ }^{1} \mathrm{H}$ $\operatorname{RMN}\left(\mathrm{CDCl}_{3}, 500 \mathrm{MHz}, \mathrm{ppm}\right) \delta=5.62(\mathrm{brs}, 1 \mathrm{H}), 3.82-3.75(\mathrm{~m}, 1 \mathrm{H}), 3.51(\mathrm{t}, J=9 \mathrm{~Hz}, 1 \mathrm{H})$, 3.42-3.32 (m, 2H), $3.14(\mathrm{dd}, J=5 \mathrm{~Hz}, 9 \mathrm{~Hz}, 1 \mathrm{H}), 2.76(\mathrm{~s}, 3 \mathrm{H}) ;{ }^{13} \mathrm{C} \mathrm{RMN}\left(\mathrm{CDCl}_{3}, 125 \mathrm{MHz}\right.$, ppm) $\delta=161.9,55.0,50.5,49.1,30.5 ; \mathrm{IR}\left(v=\mathrm{cm}^{-1}\right): 3274,2930,2868,2103,1697,1502$, 1275; MS (ESI): $\mathrm{m} / \mathrm{z}=156.17\left[\mathrm{M}+\mathrm{H}^{+}\right]$; HRMS (ESI): $\mathrm{m} / \mathrm{z}$ calcd for $\mathrm{C}_{5} \mathrm{H}_{10} \mathrm{~N}_{5} \mathrm{O}\left[\mathrm{M}+\mathrm{H}^{+}\right]$ 156.08799, found 156.08784 .

2c - 4-(azido(phenyl)methyl)-1-methylimidazolidin-2-one (stereochemistry was assigned by analogy with the related carbamate product)

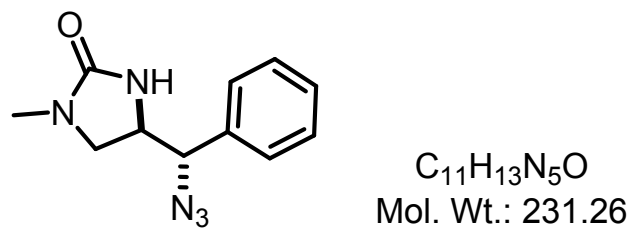

White solid; 50 mg, 75 \% Yield (Procedure B using substrate 1c 72 mg); d.r. = 93:7; m.p. 94$96{ }^{\circ} \mathrm{C}$; major diastereoisomer: ${ }^{1} \mathrm{H} \mathrm{RMN}\left(\mathrm{CDCl}_{3}, 500 \mathrm{MHz}, \mathrm{ppm}\right) \delta=7.46-7.38(\mathrm{~m}, 3 \mathrm{H}), 7.35$ $7.30(\mathrm{~m}, 2 \mathrm{H}), 4.42$ (d, $J=8.5 \mathrm{~Hz}, 1 \mathrm{H}), 4.15$ (brs, 1H), 3.77 (dt, $J=9 \mathrm{~Hz}, 5 \mathrm{~Hz}, 1 \mathrm{H}), 3.56$ (t, $J$ $=9 \mathrm{~Hz}, 1 \mathrm{H}), 3.41(\mathrm{dd}, J=5.5 \mathrm{~Hz}, 9.5 \mathrm{~Hz}, 1 \mathrm{H}), 2.78(\mathrm{~s}, 3 \mathrm{H}) ;{ }^{13} \mathrm{C} \mathrm{RMN}\left(\mathrm{CDCl}_{3}, 125 \mathrm{MHz}\right.$, ppm) $\delta=161.2,135.7,129.5,129.4,127.8,68.8,53.9,50.7,30.5$; IR $\left(v=\mathrm{cm}^{-1}\right): 3212,2096$, 2924, 2853, 2101, 1691, 1274, 1244, 701; MS (ESI): m/z = 232.25 [M+H']; HRMS (ESI): $\mathrm{m} / \mathrm{z}$ calcd for $\mathrm{C}_{11} \mathrm{H}_{14} \mathrm{~N}_{5} \mathrm{O}[\mathrm{M}+\mathrm{H}]^{+} 232.11929$, found 232.11900 . 


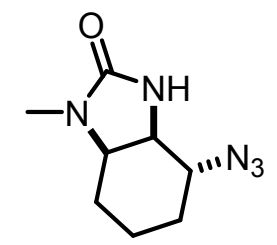

$$
\mathrm{C}_{8} \mathrm{H}_{13} \mathrm{~N}_{5} \mathrm{O}
$$

Mol. Wt.: 195.23

Colourless oil; $17 \mathrm{mg}, 30 \%$ Yield (Procedure B using substrate 1d $62 \mathrm{mg}$ ); d.r. $>95: 5 ;{ }^{1} \mathrm{H}$ $\mathrm{RMN}\left(\mathrm{CDCl}_{3}, 500 \mathrm{MHz}, \mathrm{ppm}\right) \delta=5.11(\mathrm{brs}, 1 \mathrm{H}), 3.58-3.53(\mathrm{~m}, 1 \mathrm{H}), 3.37-3.30(\mathrm{~m}, 1 \mathrm{H}), 3.16$ (t, $J=7.5 \mathrm{~Hz}, 1 \mathrm{H}), 2.69$ (s, 3H), 2.04-1.95 (m, 2H), 1.74-1.67 (m, 1H), 1.62-1.53 (m, 1H), 1.44 (tq, $J=3 \mathrm{~Hz}, 12 \mathrm{~Hz}, 1 \mathrm{H}), 1.34$ (dq, $J=3 \mathrm{~Hz}, 12 \mathrm{~Hz}, 1 \mathrm{H}) ;{ }^{13} \mathrm{C} \mathrm{RMN}\left(\mathrm{CDCl}_{3}, 125 \mathrm{MHz}\right.$, ppm) $\delta=163.1,64.0,57.9,55.2,28.5,27.5,24.6,18.7$; IR $\left(v=\mathrm{cm}^{-1}\right): 3241,2069,2940$, 2864, 2099, 1702, 1444, 1433, 1256; MS (ESI): m/z 196.3 [M+H+H]; HRMS (ESI): m/z calcd for $\mathrm{C}_{8} \mathrm{H}_{14} \mathrm{~N}_{5} \mathrm{O}[\mathrm{M}+\mathrm{H}]^{+}$196.11929, found 196.12019.

\section{2e - 4-(2-azidopropan-2-yl)-1-methylimidazolidin-2-one}

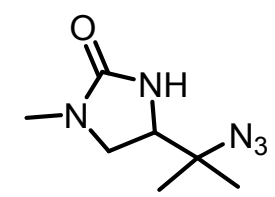

\section{$\mathrm{C}_{7} \mathrm{H}_{13} \mathrm{~N}_{5} \mathrm{O}$}

Mol. Wt.: 183.22

White solid; $33 \mathrm{mg}$, $62 \%$ Yield (Procedure B using substrate 1e $58 \mathrm{mg}$ ); m.p. $116-118{ }^{\circ} \mathrm{C} ;{ }^{1} \mathrm{H}$ RMN ( $\left.\mathrm{CDCl}_{3}, 500 \mathrm{MHz}, \mathrm{ppm}\right) \delta=5.62(\mathrm{brs}, 1 \mathrm{H}), 3.55(\mathrm{dd}, J=6.5 \mathrm{~Hz}, 9.5 \mathrm{~Hz}, 1 \mathrm{H}), 3.42(\mathrm{t}, J$ $=9.5 \mathrm{~Hz}, 1 \mathrm{H}), 3.23(\mathrm{dd}, J=6.5 \mathrm{~Hz}, 9.5 \mathrm{~Hz}, 1 \mathrm{H}), 2.76(\mathrm{~s}, 3 \mathrm{H}), 1.28(\mathrm{~s}, 3 \mathrm{H}), 1.24(\mathrm{~s}, 3 \mathrm{H}) ;{ }^{13} \mathrm{C}$ $\mathrm{RMN}\left(\mathrm{CDCl}_{3}, 125 \mathrm{MHz}, \mathrm{ppm}\right) \delta=162.0,62.9,57.3,48.7,30.4,22.1,21.5 ; \mathrm{IR}\left(\mathrm{v}=\mathrm{cm}^{-1}\right)$ : 3218, 2091, 2980, 2929, 2877, 2095, 1684, 1515, 1269; MS (ESI): $\mathrm{m} / \mathrm{z}=184.28\left[\mathrm{M}+\mathrm{H}^{+}\right]$; HRMS (ESI): $\mathrm{m} / \mathrm{z}$ calcd for $\mathrm{C}_{7} \mathrm{H}_{14} \mathrm{~N}_{5} \mathrm{O}[\mathrm{M}+\mathrm{H}]^{+} 184.11929$, found 184.11920 .

\section{2f - 4-(azidomethyl)-1,4-dimethylimidazolidin-2-one}<smiles>CN1CC(C)(CN)NC1=O</smiles>

\section{$\mathrm{C}_{6} \mathrm{H}_{11} \mathrm{~N}_{5} \mathrm{O}$}

Mol. Wt.: 169.19

Colourless oil; $26 \mathrm{mg}, 53 \%$ Yield (Procedure B using substrate 1f $54 \mathrm{mg}$ ); ${ }^{1} \mathrm{H} \mathrm{RMN}\left(\mathrm{CDCl}_{3}\right.$, $500 \mathrm{MHz}, \mathrm{ppm}) \delta=4.90$ (brs, 1H), $3.36(\mathrm{~d}, J=12 \mathrm{~Hz}, 1 \mathrm{H}), 3.29$ (d, $J=11.5 \mathrm{~Hz}, 1 \mathrm{H}), 3.27$ (d, $J=9 \mathrm{~Hz}, 1 \mathrm{H}), 3.10$ (d, $J=9.5 \mathrm{~Hz}, 1 \mathrm{H}), 2.77$ (s, 3H), 1.32 (s, 3H); ${ }^{13} \mathrm{C} \mathrm{RMN}\left(\mathrm{CDCl}_{3}, 125\right.$ MHz, ppm) $\delta=161.0,59.4,56.8,54.8,30.5,24.7$; IR $\left(v=\mathrm{cm}^{-1}\right): 3262,2973,2931,2867$, 2103, 1698, 1502, 1297, 1261, 1032, 763; MS (ESI): m/z 170.30 [M+H+]; HRMS (ESI): m/z calcd for $\mathrm{C}_{6} \mathrm{H}_{12} \mathrm{~N}_{5} \mathrm{O}[\mathrm{M}+\mathrm{H}]^{+} 170.10364$, found 170.10445 . 
4a - 4-(azidomethyl)oxazolidin-2-one<smiles>NCC1COC(=O)N1</smiles>

$$
\begin{gathered}
\mathrm{C}_{4} \mathrm{H}_{6} \mathrm{~N}_{4} \mathrm{O}_{2} \\
\text { Mol. Wt.: } 142.12
\end{gathered}
$$

White solid; $36 \mathrm{mg}, 87$ \% Yield (Procedure B using substrate 3a $46 \mathrm{mg}$ ); m.p. $68-70{ }^{\circ} \mathrm{C}$; ${ }^{1} \mathrm{H}$ $\operatorname{RMN}\left(\mathrm{CDCl}_{3}, 500 \mathrm{MHz}, \mathrm{ppm}\right) \delta=6.42(\mathrm{brs}, 1 \mathrm{H}), 4.87(\mathrm{t}, J=9 \mathrm{~Hz}, 1 \mathrm{H}), 4.17(\mathrm{dd}, J=5 \mathrm{~Hz}, 9$ $\mathrm{Hz}, 1 \mathrm{H}), 4.05-3.98(\mathrm{~m}, 1 \mathrm{H}), 3.51$ (dd, $J=5 \mathrm{~Hz}, 12.5 \mathrm{~Hz}, 1 \mathrm{H}), 3.43$ (dd, $J=5.5 \mathrm{~Hz}, 12 \mathrm{~Hz}$, $1 \mathrm{H}) ;{ }^{13} \mathrm{C} \mathrm{RMN}\left(\mathrm{CDCl}_{3}, 125 \mathrm{MHz}, \mathrm{ppm}\right) \delta=159.7,67.6,54.2,51.8 ; \mathrm{IR}\left(v=\mathrm{cm}^{-1}\right): 3671$, 3212, 3122, 2987, 2923, 294, 1726, 1242, 1008, 698; MS (ESI): m/z = $165.03\left[\mathrm{M}+\mathrm{Na}^{+}\right]$; HRMS (ESI): $\mathrm{m} / \mathrm{z}$ calcd for $\mathrm{C}_{4} \mathrm{H}_{7} \mathrm{~N}_{4} \mathrm{O}_{2}\left[\mathrm{M}+\mathrm{H}^{+}\right]$143.05635, found 143.05612 .

\section{4b - 4-(azido(phenyl)methyl)oxazolidin-2-one (known compound) 5}<smiles>N[C@H](c1ccccc1)C1COC(=O)N1</smiles>

\section{$\mathrm{C}_{10} \mathrm{H}_{10} \mathrm{~N}_{4} \mathrm{O}_{2}$}

Mol. Wt.: 218.22

White solid; 57 mg, 90 \% Yield (Procedure B using substrate 3b 68 mg); dr > 98:2; m.p. 80$82{ }^{\circ} \mathrm{C} ;{ }^{1} \mathrm{H} \mathrm{RMN}\left(\mathrm{CDCl}_{3}, 500 \mathrm{MHz}, \mathrm{ppm}\right) \delta=7.48-7.40$ (m, 3H), 7.36-7.31 (m, 2H), 5.18 (brs, 1H), 4.52 (d, $J=8 \mathrm{~Hz}, 1 \mathrm{H}), 4.47$ (dd, $J=8 \mathrm{~Hz}, 9 \mathrm{~Hz}, 1 \mathrm{H}), 4.39$ (dd, $J=5 \mathrm{~Hz}, 9 \mathrm{~Hz}, 1 \mathrm{H}), 3.98$ (tdd, $J=1 \mathrm{~Hz}, 5 \mathrm{~Hz}, 8.5 \mathrm{~Hz}, 1 \mathrm{H}) ;{ }^{13} \mathrm{C} \mathrm{RMN}\left(\mathrm{CDCl}_{3}, 125 \mathrm{MHz}, \mathrm{ppm}\right) \delta=158.7,134.9,129.8$, 129.7, 127.6, 68.3, 67.7, 56.5; IR $\left(v=\mathrm{cm}^{-1}\right): 3232,3136,2926,2105,1731,1241,1033,1024$; MS (ESI): $\mathrm{m} / \mathrm{z}=219.25\left[\mathrm{M}+\mathrm{H}^{+}\right]$; HRMS (ESI): $\mathrm{m} / \mathrm{z}$ calcd for $\mathrm{C}_{10} \mathrm{H}_{11} \mathrm{~N}_{4} \mathrm{O}_{2}\left[\mathrm{M}+\mathrm{H}^{+}\right]$ 219.08765 , found 219.08745 .

\section{4c - 4-(azido(4-chlorophenyl)methyl)oxazolidin-2-one (known compound) ${ }^{5}$}

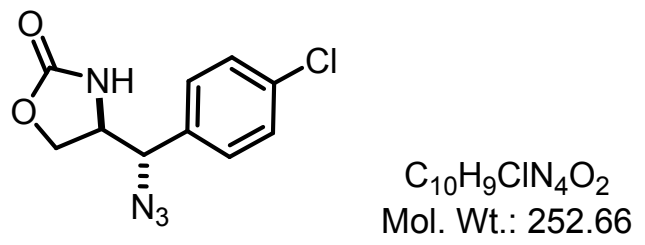

White solid; $58 \mathrm{mg}, 85 \%$ Yield (Procedure B using substrate 3c $73 \mathrm{mg}$ ); dr > 98:2; m.p. 112 ${ }^{\circ} \mathrm{C} ;{ }^{1} \mathrm{H} \mathrm{RMN}\left(\mathrm{CDCl}_{3}, 500 \mathrm{MHz}, \mathrm{ppm}\right) \delta=7.42(\mathrm{~d}, J=8.5 \mathrm{~Hz}, 2 \mathrm{H}), 7.28(\mathrm{~d}, J=8 \mathrm{~Hz}, 2 \mathrm{H})$, 5.98 (brs, 1H), 4.57 (d, $J=7.5 \mathrm{~Hz}, 1 \mathrm{H}), 4.40(\mathrm{dd}, J=8.5 \mathrm{~Hz}, 9.5 \mathrm{~Hz}, 1 \mathrm{H}), 4.32$ (dd, $J=4.5$ $\mathrm{Hz}, 9 \mathrm{~Hz}, 1 \mathrm{H}), 4.01-3.95(\mathrm{~m}, 1 \mathrm{H}) ;{ }^{13} \mathrm{C} \mathrm{RMN}\left(\mathrm{CDCl}_{3}, 125 \mathrm{MHz}, \mathrm{ppm}\right) \delta=159.2,135.6,133.4$, $129.8,128.9,67.4,67.2,56.6$; IR $\left(v=\mathrm{cm}^{-1}\right): 3403,2987,2915,2102,1784,1750,1212,1017$; 
MS (ESI): $\mathrm{m} / \mathrm{z}=250.92\left[\mathrm{M}-\mathrm{H}^{+}\right] ; \operatorname{HRMS}(\mathrm{ESI}): \mathrm{m} / \mathrm{z}$ calcd for $\mathrm{C}_{10} \mathrm{H}_{8} \mathrm{ClN}_{4} \mathrm{O}_{2} \quad\left[\mathrm{M}-\mathrm{H}^{+}\right]$ 251.03413 , found 251.03398 .

4d - 4-(azido(4-nitrophenyl)methyl)oxazolidin-2-one (stereochemistry was assigned by analogy with the other products)<smiles>N[C@@H](c1ccc([N+](=O)[O-])cc1)[C@H]1COC(=O)N1</smiles>

Pale yellow solid; 57 mg, 75 \% (Procedure B using substrate 3d $82 \mathrm{mg}$ ); dr = 95:5; m.p. 124$126{ }^{\circ} \mathrm{C}$; major diastereoisomer : ${ }^{1} \mathrm{H} \mathrm{RMN}\left(\mathrm{CDCl}_{3}, 500 \mathrm{MHz}, \mathrm{ppm}\right) \delta=8.32(\mathrm{~d}, J=9 \mathrm{~Hz}, 2 \mathrm{H})$, $7.56(\mathrm{~d}, J=8.5 \mathrm{~Hz}, 2 \mathrm{H}), 5.73$ (brs, 1H), $4.70(\mathrm{~d}, J=7 \mathrm{~Hz}, 1 \mathrm{H}), 4.45$ (t, $J=9 \mathrm{~Hz}, 1 \mathrm{~Hz}, 1 \mathrm{H})$, $4.36(\mathrm{dd}, J=3.5 \mathrm{~Hz}, 9.5 \mathrm{~Hz}, 1 \mathrm{H}), 4.07-4.00(\mathrm{~m}, 1 \mathrm{H}) ;{ }^{13} \mathrm{C} \mathrm{RMN}\left(\mathrm{CDCl}_{3}, 125 \mathrm{MHz}, \mathrm{ppm}\right) \delta=$ 158.8, 148.8, 142.0, 128.6, 124.8, 67.4, 67.2, 56.5; IR $\left(v=\mathrm{cm}^{-1}\right): 3142,3116,2977,2899$, 2120, 1750, 1517, 1350, 744; HRMS (ESI): m/z calcd for $\mathrm{C}_{10} \mathrm{H}_{8} \mathrm{~N}_{5} \mathrm{O}_{4}\left[\mathrm{M}-\mathrm{H}^{+}\right] 262.05818$, found 262.05859 .

\section{4e - 4-(azido(2-methoxyphenyl)methyl)oxazolidin-2-one (known compound) 5}

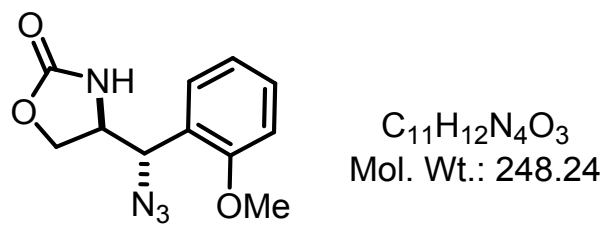

The crude NMR shown the presence of the corresponding acetylated product in nonnegligible amount. In this case to remove this by-product a supplementary workup was needed. After procedure B workup, the crude was filtrated on silica gel and the solvent removed under vacuum. Then the residue was taken on $2 \mathrm{~mL}$ of $\mathrm{MeOH}$ and $10 \mathrm{mg}$ of $\mathrm{K}_{2} \mathrm{CO}_{3}$ was added. The mixture was stirred at $0{ }^{\circ} \mathrm{C}$ for $30 \mathrm{mn}$. The solvent was removed and the residue purified by silica gel flash chromatography using Cyclohexane and AcOEt as eluent (from 70/30 to 50/50 ratio).

White solid; $39 \mathrm{mg}, 54 \%$ Yield (modified procedure B as above using substrate $3 \mathrm{e} 77 \mathrm{mg}$ ); $\mathrm{dr}$ > 98:2; m.p. $112-114{ }^{\circ} \mathrm{C} ;{ }^{1} \mathrm{H} \mathrm{RMN}\left(\mathrm{CDCl}_{3}, 500 \mathrm{MHz}, \mathrm{ppm}\right) \delta=7.38$ (td, $J=1.5 \mathrm{~Hz}, 8.5 \mathrm{~Hz}$, 1H), $7.32(\mathrm{dd}, J=1.5 \mathrm{~Hz}, 7.5 \mathrm{~Hz}, 1 \mathrm{H}), 7.05(\mathrm{t}, J=7.5 \mathrm{~Hz}, 1 \mathrm{H}), 6.97$ (d, $J=8 \mathrm{~Hz}, 1 \mathrm{H}), 5.04$ (d, $J=7.5 \mathrm{~Hz}, 1 \mathrm{H}), 4.90$ (brs, $1 \mathrm{H}), 4.43-4.34$ (m, 2H), 4.18-4.12 (m, 1H), $3.87(\mathrm{~s}, 3 \mathrm{H}) ;{ }^{13} \mathrm{C}$ $\mathrm{RMN}\left(\mathrm{CDCl}_{3}, 125 \mathrm{MHz}, \mathrm{ppm}\right) \delta=158.8,157.2,130.8,128.2,122.8,121.5,111.3,67.5,62.2$, 
55.8, 54.9; IR $\left(v=\mathrm{cm}^{-1}\right): 3231,2970,2915,2837,2118,1741,1724,1248,1236,1029,1020$, 757; MS (ESI): $\mathrm{m} / \mathrm{z}=249.20\left[\mathrm{M}+\mathrm{H}^{+}\right] ; \operatorname{HRMS}(\mathrm{ESI}): \mathrm{m} / \mathrm{z}$ calcd for $\mathrm{C}_{11} \mathrm{H}_{13} \mathrm{~N}_{4} \mathrm{O}_{3}\left[\mathrm{M}+\mathrm{H}^{+}\right]$ 249.09931 , found 249.09782 .

\title{
4f - 4-(azido(4-methoxyphenyl)methyl)oxazolidin-2-one (known compound) ${ }^{5}$
}<smiles>COc1ccc(C(N)C2COC(=O)N2)cc1</smiles>

Sticky yellow paste; $16 \mathrm{mg}, 22 \%$ Yield (Procedure B using substrate $3 f 77 \mathrm{mg}$ ); dr = 50:50; mixture of diastereoisomers: ${ }^{1} \mathrm{H} \mathrm{RMN}\left(\mathrm{CDCl}_{3}, 500 \mathrm{MHz}, \mathrm{ppm}\right) \delta=7.25$ (t, $\left.J=8 \mathrm{~Hz}, 4 \mathrm{H}\right)$, 7.00-6.94 (m, 4H), 5.96 (brs, 1H), 5.35 (brs, 1H), 4.51-4.43 (m, 3H), 4.36 (dd, $J=4.5 \mathrm{~Hz}, 9$ $\mathrm{Hz}, 1 \mathrm{H}), 4.22-4.15(\mathrm{~m}, 1 \mathrm{H}), 4.03-3.94(\mathrm{~m}, 3 \mathrm{H}), 3.84(\mathrm{~s}, 3 \mathrm{H}), 3.83(\mathrm{~s}, 3 \mathrm{H}) ;{ }^{13} \mathrm{C} \mathrm{RMN}\left(\mathrm{CDCl}_{3}\right.$, $125 \mathrm{MHz}, \mathrm{ppm}) \delta=160.7,160.6,158.9,129.1,128.9,126.7,126.1,115.0,69.0,67.8,67.7$, 66.7, 56.7, 56.4, 55.5; IR $\left(v=\mathrm{cm}^{-1}\right): 3302,2966,2837,2107,1753,1514,1248,1030 ; \mathrm{MS}$ (ESI): $\mathrm{m} / \mathrm{z}=271.25\left[\mathrm{M}+\mathrm{Na}^{+}\right]$; HRMS (ESI): $\mathrm{m} / \mathrm{z}$ calcd for $\mathrm{C}_{11} \mathrm{H}_{13} \mathrm{~N}_{4} \mathrm{O}_{3}\left[\mathrm{M}+\mathrm{H}^{+}\right]$249.09822, found 249.09782 .

4g - 4-(azidomethyl)-4-methyloxazolidin-2-one<smiles>CC1(CN)COC(=O)N1</smiles>

\author{
$\mathrm{C}_{5} \mathrm{H}_{8} \mathrm{~N}_{4} \mathrm{O}_{2}$ \\ Mol. Wt.: 156.15
}

Colourless oil; $28.5 \mathrm{mg}, 63 \%$ Yield (Procedure B using substrate $3 \mathrm{~g} 50 \mathrm{mg}$ ); ${ }^{1} \mathrm{H}$ RMN $\left(\mathrm{CDCl}_{3}, 500 \mathrm{MHz}, \mathrm{ppm}\right) \delta=6.75(\mathrm{brs}, 1 \mathrm{H}), 4.25(\mathrm{~d}, J=9 \mathrm{~Hz}, 1 \mathrm{H}), 4.02(\mathrm{~d}, J=9 \mathrm{~Hz}, 1 \mathrm{H})$, $3.45(\mathrm{~d}, J=12.5,1 \mathrm{H}), 3.32(\mathrm{~d}, J=12.5,1 \mathrm{H}), 1.37(\mathrm{~s}, 3 \mathrm{H}) ;{ }^{13} \mathrm{C} \mathrm{RMN}\left(\mathrm{CDCl}_{3}, 125 \mathrm{MHz}, \mathrm{ppm}\right)$ $\delta=159.3,73.4,58.5,58.1,23.7 ;$ IR $\left(v=\mathrm{cm}^{-1}\right): 3278,2979,2917,2105,1742,1244,1042$; MS (ESI): $\mathrm{m} / \mathrm{z}=157.16\left[\mathrm{M}+\mathrm{H}^{+}\right]$; HRMS (ESI): $\mathrm{m} / \mathrm{z}$ calcd for $\mathrm{C}_{5} \mathrm{H}_{9} \mathrm{~N}_{4} \mathrm{O}_{2}\left[\mathrm{M}+\mathrm{H}^{+}\right] 157.07200$, found 157.07179 .

\section{4h - 4-(azido(phenyl)methyl)-4-methyloxazolidin-2-one}




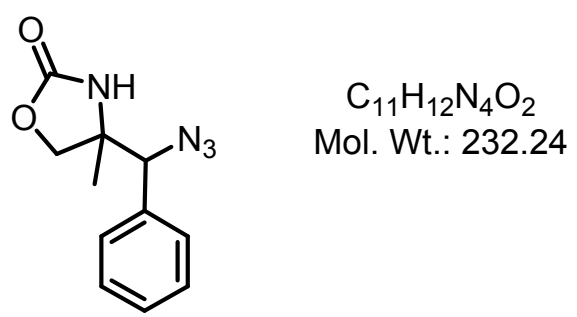

White solid; $44 \mathrm{mg}, 65 \%$ Yield (Procedure B using substrate $3 \mathbf{h ~} 72 \mathrm{mg}$ ); d.r. $=60: 40$; m..p. 96-98 ${ }^{\circ} \mathrm{C}$; mixture of diastereoisomers: ${ }^{1} \mathrm{H} \mathrm{RMN}\left(\mathrm{CDCl}_{3}, 500 \mathrm{MHz}, \mathrm{ppm}\right) \delta=7.45-7.37$ (m, $5 \mathrm{H}_{\text {dia maj) }}$ ), 7.37-7.30 (m, 3.5 $\mathrm{H}_{\text {dia min }}$ ), 6.57 (brs, $0.7 \mathrm{H}_{\text {dia min }}$ ), 6.22 (brs, $\left.1 \mathrm{H}_{\text {dia maj }}\right), 4.63$ (s, $1 \mathrm{H}$ dia maj), 4.60 (s, $0.7_{\text {dia min }}$ ), 4.45 (d, $\left.J=9 \mathrm{~Hz}, 1 \mathrm{H}_{\text {dia maj) }}\right), 4.44$ (d, $J=9 \mathrm{~Hz}, 0.7$ dia min $), 3.98$ (d, $J$ $\left.=9 \mathrm{~Hz}, 1 \mathrm{H}_{\text {dia maj }}\right), 3.89\left(\mathrm{~d}, J=9 \mathrm{~Hz}, 0.7 \mathrm{H}_{\text {dia min }}\right), 1.30\left(\mathrm{~s}, 2.1 \mathrm{H}_{\text {dia min }}\right), 1.28\left(\mathrm{~s}, 3 \mathrm{H}_{\text {dia maj }}\right) ;{ }^{13} \mathrm{C}$ $\mathrm{RMN}\left(\mathrm{CDCl}_{3}, 125 \mathrm{MHz}, \mathrm{ppm}\right) \delta=158.9,158.8,134.3,129.3,129.0,128.2,128.1,73.2,72.7$, 71.8, 71.5, 60.7, 60.6, 23.0, 22.6; IR $\left(v=\mathrm{cm}^{-1}\right): 3675,3273,2988,2901,2109,1734,1722$, 1258, 1043, 706; MS (ESI): $\mathrm{m} / \mathrm{z}=233.15\left[\mathrm{M}+\mathrm{H}^{+}\right]$; HRMS (ESI): $\mathrm{m} / \mathrm{z}$ calcd for $\mathrm{C}_{11} \mathrm{H}_{13} \mathrm{~N}_{4} \mathrm{O}_{2}$ $\left[\mathrm{M}+\mathrm{H}^{+}\right]$233.10440, found 233.10300.

\section{4i - 4-azidohexahydrobenzo[d]oxazol-2(3H)-one (known compound) ${ }^{6}$}<smiles>N[C@H]1CCCC2OC(=O)NC21</smiles>

$$
\mathrm{C}_{7} \mathrm{H}_{10} \mathrm{~N}_{4} \mathrm{O}_{2}
$$

Mol. Wt.: 182.18

White solid; $26 \mathrm{mg}, 50$ \% Yield (Procedure B using substrate 3i $58 \mathrm{mg}$ ); d.r. > 98:2; m.p. 84 ${ }^{\circ} \mathrm{C} ;{ }^{1} \mathrm{H} \mathrm{RMN}\left(\mathrm{CDCl}_{3}, 500 \mathrm{MHz}, \mathrm{ppm}\right) \delta=5.37$ (brs, $\left.1 \mathrm{H}\right), 4.73-4.68(\mathrm{~m}, 1 \mathrm{H}), 3.44-3.38$ (m, $1 \mathrm{H}), 3.32$ (dd, $J=6 \mathrm{~Hz}, 8.5 \mathrm{~Hz}, 1 \mathrm{H}), 2.27-2.20(\mathrm{~m}, 1 \mathrm{H}), 2.11-2.03(\mathrm{~m}, 1 \mathrm{H}), 1.83-1.75$ (m, $1 \mathrm{H}), 1,72-1.59(\mathrm{~m}, 2 \mathrm{H}), 1.42-1.30(\mathrm{~m}, 1 \mathrm{H}) ;{ }^{13} \mathrm{C} \mathrm{RMN}\left(\mathrm{CDCl}_{3}, 125 \mathrm{MHz}, \mathrm{ppm}\right) \delta=159.1$, 76.6, 64.1, 57.2, 26.7, 26.2, 18.5; IR $\left(v=\mathrm{cm}^{-1}\right): 3310,2960,2883,2116,2095,1739,1226$; MS (ESI): $\mathrm{m} / \mathrm{z}=183.21\left[\mathrm{M}+\mathrm{H}^{+}\right]$; HRMS (ESI): $\mathrm{m} / \mathrm{z}$ calcd for $\mathrm{C}_{7} \mathrm{H}_{11} \mathrm{~N}_{4} \mathrm{O}_{2}\left[\mathrm{M}+\mathrm{H}^{+}\right] 183.08765$, found 183.08746 .

\section{4j - 4-(2-azidopropan-2-yl)oxazolidin-2-one}

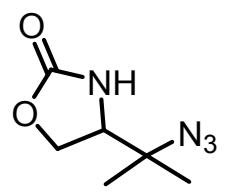

$$
\begin{gathered}
\mathrm{C}_{6} \mathrm{H}_{10} \mathrm{~N}_{4} \mathrm{O}_{2} \\
\text { Mol. Wt.: } 170.17
\end{gathered}
$$

The crude NMR shown the presence of the corresponding acetylated product in nonnegligible amount. In this case to remove this by-product a supplementary workup was 
needed. After procedure B workup, the crude was filtrated on silica gel and the solvent removed under vacuum. Then the residue was taken on $2 \mathrm{~mL}$ of $\mathrm{MeOH}$ and $10 \mathrm{mg}$ of $\mathrm{K}_{2} \mathrm{CO}_{3}$ was added. The mixture was stirred at $0{ }^{\circ} \mathrm{C}$ for $30 \mathrm{mn}$. The solvent was removed and the residue purified by silica gel flash chromatography using Cyclohexane and AcOEt as eluent (from 70/30 to 50/50 ratio).

White solid; $21 \mathrm{mg}, 43 \%$ Yield (modified procedure B see above using substrate $3 \mathbf{j} 54 \mathrm{mg}$ ) (30\% Yield in pure ACN); m.p. $80{ }^{\circ} \mathrm{C} ;{ }^{1} \mathrm{H} \mathrm{RMN}\left(\mathrm{CDCl}_{3}, 500 \mathrm{MHz}, \mathrm{ppm}\right) \delta=5.66$ (brs, $\left.1 \mathrm{H}\right)$, $4.42(\mathrm{t}, J=9 \mathrm{~Hz}, 1 \mathrm{H}), 4.25$ (dd, $J=5 \mathrm{~Hz}, 9.5 \mathrm{~Hz}, 1 \mathrm{H}), 3.72$ (ddd, $J=1.5 \mathrm{~Hz}, 5 \mathrm{~Hz}, 9 \mathrm{~Hz}, 1 \mathrm{H})$, $1.33(\mathrm{~s}, 3 \mathrm{H}), 1,30(\mathrm{~s}, 3 \mathrm{H}) ;{ }^{13} \mathrm{C} \mathrm{RMN}\left(\mathrm{CDCl}_{3}, 125 \mathrm{MHz}, \mathrm{ppm}\right) \delta=159.2,66.1,62.5,59.7$, 21.6, 21.5; IR $\left(v=\mathrm{cm}^{-1}\right): 3242,3139,2976,2112,2096,1736,1271,1248$; MS (ESI): $\mathrm{m} / \mathrm{z}=$ $171.25\left[\mathrm{M}+\mathrm{H}^{+}\right]$; HRMS (ESI): $\mathrm{m} / \mathrm{z}$ calcd for $\mathrm{C}_{6} \mathrm{H}_{11} \mathrm{~N}_{4} \mathrm{O}_{2}\left[\mathrm{M}+\mathrm{H}^{+}\right] 171.08765$, found 171.08740 .

\title{
6a - 5-(azidomethyl)pyrrolidin-2-one (known compound) ${ }^{7}$
}

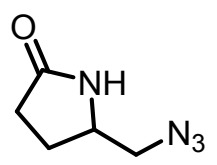

\author{
$\mathrm{C}_{5} \mathrm{H}_{8} \mathrm{~N}_{4} \mathrm{O}$ \\ Mol. Wt.: 140.15
}

Pale red oil; $29 \mathrm{mg}, 71 \%$ Yield (Procedure B using substrate 5a $46 \mathrm{mg}) ;{ }^{1} \mathrm{H} \mathrm{RMN}\left(\mathrm{CDCl}_{3}\right.$, $500 \mathrm{MHz}, \mathrm{ppm}) \delta=6.48$ (brs, $1 \mathrm{H}), 3.86-3.77$ (m, 1H), 3.47 (dd, $J=4.5,12.5 \mathrm{~Hz}, 1 \mathrm{H}), 3.30$ $(\mathrm{dd}, J=7,12.5 \mathrm{~Hz}, 1 \mathrm{H}), 2.46-2.33(\mathrm{~m}, 2 \mathrm{H}), 2.33-2.24(\mathrm{~m}, 1 \mathrm{H}), 1.87-1.78(\mathrm{~m}, 1 \mathrm{H}) ;{ }^{13} \mathrm{C} \mathrm{RMN}$ $\left(\mathrm{CDCl}_{3}, 125 \mathrm{MHz}, \mathrm{ppm}\right) \delta=178.2,56.2,53.6,29.7,24.2$; IR $\left(v=\mathrm{cm}^{-1}\right): 3659,3229,3101$, 2972, 2925, 2101, 1688, 1278; MS (ESI): m/z 141.3 [M+H'] ; HRMS (ESI): m/z calcd for $\mathrm{C}_{5} \mathrm{H}_{9} \mathrm{~N}_{4} \mathrm{O}[\mathrm{M}+\mathrm{H}]^{+}$141.07709, found 141.07787.

The stereochemistry of the disubstituted products was assigned by analogy with the other products.

\section{6b - 5-(azido(phenyl)methyl)pyrrolidin-2-one}

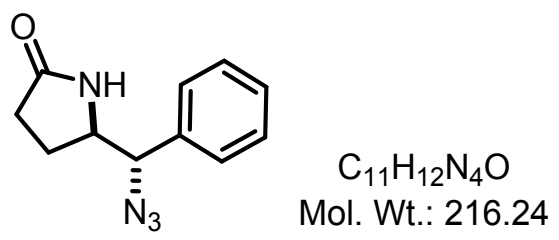

Colourless oil; 34 mg, 54 \% Yield (Procedure B using substrate 5b 68 mg); d.r. = 70:30; major diastereoisomer: ${ }^{1} \mathrm{H} \mathrm{RMN}\left(\mathrm{CDCl}_{3}, 500 \mathrm{MHz}, \mathrm{ppm}\right) \delta=7.46-7.36(\mathrm{~m}, 3 \mathrm{H}), 7.35-7.29$ (m, 2H), 5.91 (brs, 1H), 4.44 (d, $J=7 \mathrm{~Hz}, 1 \mathrm{H}), 3.90-3.80$ (m, 1H), 2.34-2.14 (m, 3H), $2.07-$ 
$1.98(\mathrm{~m}, 1 \mathrm{H}) ;{ }^{13} \mathrm{C} \mathrm{RMN}\left(\mathrm{CDCl}_{3}, 125 \mathrm{MHz}, \mathrm{ppm}\right) \delta=178.2,135.7,129.3,127.7,69.6,58.3$, 29.5, 23.7; IR $\left(v=\mathrm{cm}^{-1}\right): 3214,3090,3028,2926,2102,1693,1250,702 ;$ MS (ESI): $\mathrm{m} / \mathrm{z}$ $217.2\left[\mathrm{M}+\mathrm{H}^{+}\right]$; HRMS (ESI): $\mathrm{m} / \mathrm{z}$ calcd for $\mathrm{C}_{11} \mathrm{H}_{13} \mathrm{~N}_{4} \mathrm{O}\left[\mathrm{M}+\mathrm{H}^{+}\right] 217.10839$, found 217.10947.

\section{6c - 5-(azido(2-methoxyphenyl)methyl)pyrrolidin-2-one}

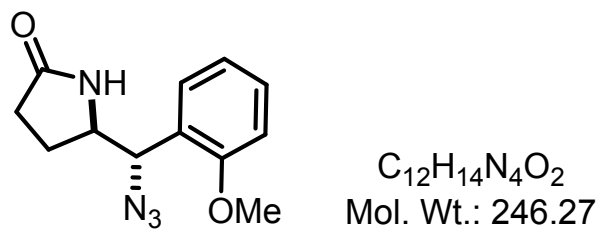

Pale red oil; 19 mg, 27 \% Yield (Procedure B using substrate 5c 76 mg); d.r. = 75:25; major diastereoisomer: ${ }^{1} \mathrm{H} \mathrm{RMN}\left(\mathrm{CDCl}_{3}, 500 \mathrm{MHz}, \mathrm{ppm}\right) \delta=7.38-7.28(\mathrm{~m}, 2 \mathrm{H}), 7.06-7.00(\mathrm{~m}, 1 \mathrm{H})$, 6.95 (d, $J=8.5 \mathrm{~Hz}, 1 \mathrm{H}), 5.93$ (brs, 1H), 4.96 (d, $J=7.5 \mathrm{~Hz}, 1 \mathrm{H}), 3.94-3.88$ (m, 1H), 3.86 (s, $3 \mathrm{H}), 2.43-2.32(\mathrm{~m}, 1 \mathrm{H}), 2.31-2.23(\mathrm{~m}, 1 \mathrm{H}), 2.07-1.98(\mathrm{~m}, 1 \mathrm{H}), 1.89-1.76(\mathrm{~m}, 1 \mathrm{H}) ;{ }^{13} \mathrm{C} \mathrm{RMN}$ $\left(\mathrm{CDCl}_{3}, 125 \mathrm{MHz}, \mathrm{ppm}\right) \delta=177.9,157.1,130.2,128.0,124.0,121.3,111.2,64.1,57.5,55.7$, 29.9, 23.7; IR $\left(v=\mathrm{cm}^{-1}\right): 3212,3077,2940,2840,2102,1694,1493,1247,756$; MS (ESI): $\mathrm{m} / \mathrm{z}=247.19\left[\mathrm{M}+\mathrm{H}^{+}\right]$; HRMS (ESI): $\mathrm{m} / \mathrm{z}$ calcd for $\mathrm{C}_{12} \mathrm{H}_{15} \mathrm{~N}_{4} \mathrm{O}_{2}[\mathrm{M}+\mathrm{H}]^{+}$247.11895, found 247.11847.

\section{6d - 5-(1-azidoethyl)pyrrolidin-2-one}<smiles>C[C@H](N)C1CCC(=O)N1</smiles>

\section{$\mathrm{C}_{6} \mathrm{H}_{10} \mathrm{~N}_{4} \mathrm{O}$}

Mol. Wt.: 154.17

Melting white solid; $34 \mathrm{mg}, 76$ \% Yield (Procedure B using substrate 5d $50 \mathrm{mg}$ ); d.r. = 80:20; major diastereoisomer: ${ }^{1} \mathrm{H} \mathrm{RMN}\left(\mathrm{CDCl}_{3}, 500 \mathrm{MHz}, \mathrm{ppm}\right) \delta=7.18$ (brs, $\left.1 \mathrm{H}\right), 3.62$ (dt, $J=5$ $\mathrm{Hz}, 8 \mathrm{~Hz}, 1 \mathrm{H}), 3.59-3.53(\mathrm{~m}, 1 \mathrm{H}), 2.43-2.35$ (m, 1H), 2.34-2.26 (m, 1H), 2.24-2.14 (m, 1H), 1.97-1.88 (m, 1H), $1.27(\mathrm{~d}, J=6.5 \mathrm{~Hz}, 3 \mathrm{H}) ;{ }^{13} \mathrm{C} \mathrm{RMN}\left(\mathrm{CDCl}_{3}, 125 \mathrm{MHz}, \mathrm{ppm}\right) \delta=179.1$, 61.0, 58.4, 29.8, 22.6, 15.5; IR $\left(v=\mathrm{cm}^{-1}\right): 3217,2978,2934,2109,1693,1265$; MS (ESI): $\mathrm{m} / \mathrm{z}=177.32\left[\mathrm{M}+\mathrm{Na}^{+}\right]$; HRMS (ESI): $\mathrm{m} / \mathrm{z}$ calcd for $\mathrm{C}_{6} \mathrm{H}_{11} \mathrm{~N}_{4} \mathrm{O}[\mathrm{M}+\mathrm{H}]^{+} 155.09274$, found 155.09250.

6e - 5-(azidomethyl)-5-methylpyrrolidin-2-one<smiles>CC1(CN)CCC(=O)N1</smiles>

$\mathrm{C}_{6} \mathrm{H}_{10} \mathrm{~N}_{4} \mathrm{O}$ Mol. Wt.: 154.17 
Colourless oil; $33 \mathrm{mg}, 74 \%$ Yield (Procedure B using substrate 5e $50 \mathrm{mg}) ;{ }^{1} \mathrm{H}$ RMN $\left(\mathrm{CDCl}_{3}\right.$, $500 \mathrm{MHz}, \mathrm{ppm}) \delta=7.13$ (brs, $1 \mathrm{H}), 3.35$ (d, $J=12 \mathrm{~Hz}, 1 \mathrm{H}), 3.26(\mathrm{~d}, J=12.5 \mathrm{~Hz}, 1 \mathrm{H}), 2.50$ 2.35 (m, 2H), 2.02 (ddd, $J=5.5 \mathrm{~Hz}, 9.5 \mathrm{~Hz}, 13 \mathrm{~Hz}, 1 \mathrm{H}), 1.90-1.81(\mathrm{~m}, 1 \mathrm{H}), 1.30(\mathrm{~s}, 3 \mathrm{H}) ;{ }^{13} \mathrm{C}$ $\mathrm{RMN}\left(\mathrm{CDCl}_{3}, 125 \mathrm{MHz}, \mathrm{ppm}\right) \delta=177.8,60.6,59.7,31.4,30.5,25.6$; IR $\left(v=\mathrm{cm}^{-1}\right)$ : 3223, 2972, 2932, 2102, 1690, 1420, 1383, 1301, 1249; MS (ESI): m/z = $155.2\left[\mathrm{M}+\mathrm{H}^{+}\right]$; HRMS (ESI): $\mathrm{m} / \mathrm{z}$ calcd for $\mathrm{C}_{6} \mathrm{H}_{11} \mathrm{~N}_{4} \mathrm{O}\left[\mathrm{M}+\mathrm{H}^{+}\right]$155.09274, found 155.09238 .

\section{6f - 5-(azidomethyl)-4-methylpyrrolidin-2-one}<smiles>CC1CC(=O)N[C@H]1CN</smiles>

\section{$\mathrm{C}_{6} \mathrm{H}_{10} \mathrm{~N}_{4} \mathrm{O}$}

Mol. Wt.: 154.17

Colourless oil; $33 \mathrm{mg}, 74 \%$ Yield (Procedure B using substrate 5f $50 \mathrm{mg}$ ); d.r. = 65:35; mixture of diastereoisomers: ${ }^{1} \mathrm{H} \mathrm{RMN}\left(\mathrm{CDCl}_{3}, 500 \mathrm{MHz}, \mathrm{ppm}\right) \delta=7.16$ (brs, $1.5 \mathrm{H}$ dia min + dia

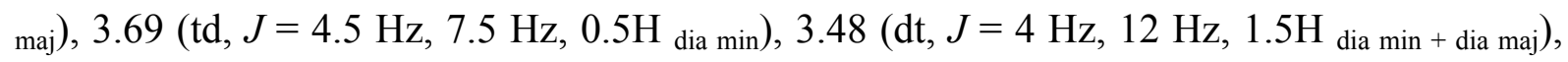

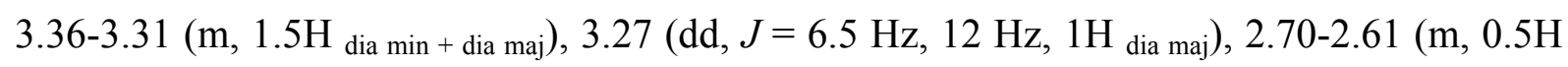
dia min), 2.56 (dd, $J=8.5 \mathrm{~Hz}, 17 \mathrm{~Hz}, 1 \mathrm{H}_{\text {dia maj) }} 2.41$ (dd, $J=8.5 \mathrm{~Hz}, 16.5 \mathrm{~Hz}, 0.5 \mathrm{H}_{\text {dia } \min }$ ), 2.23-2.14 (m, $1 \mathrm{H}_{\text {dia maj) }}$ ) 2.08 (dd, $\left.J=9 \mathrm{~Hz}, 17 \mathrm{~Hz}, 0.5 \mathrm{H}_{\text {dia } \min }\right), 1.99$ (dd, $J=6.5 \mathrm{~Hz}, 17 \mathrm{~Hz}$, $\left.1 \mathrm{H}_{\text {dia maj }}\right), 1.14$ (d, $\left.J=7.5 \mathrm{~Hz}, 3 \mathrm{H}_{\text {dia maj }}\right), 1.06$ (d, $\left.J=7.5 \mathrm{~Hz}, 1.5 \mathrm{H}_{\text {dia min }}\right) ;{ }^{13} \mathrm{C} \mathrm{RMN}\left(\mathrm{CDCl}_{3}\right.$, $125 \mathrm{MHz}, \mathrm{ppm}) \delta=178.2,177.9,61.2,56.5,55.0,52.6,38.5,38.1,32.6,31.7,19.5,14.6$; IR $\left(v=\mathrm{cm}^{-1}\right): 3229,2965,2930,2873,2103,1691,1439,1382,1306,1274 ;$ MS (ESI): $\mathrm{m} / \mathrm{z}=$ $155.2\left[\mathrm{M}+\mathrm{H}^{+}\right]$; HRMS (ESI): m/z calcd for $\mathrm{C}_{6} \mathrm{H}_{11} \mathrm{~N}_{4} \mathrm{O}[\mathrm{M}+\mathrm{H}]^{+}$155.09274, found 155.09270.

\section{7 - tert-butyl 4-(azidomethyl)-2-oxooxazolidine-3-carboxylate}<smiles>NCC1COC(=O)N1C(=O)OCc1ccccc1</smiles>

\section{$\mathrm{C}_{9} \mathrm{H}_{14} \mathrm{~N}_{4} \mathrm{O}_{4}$}

Mol. Wt.: 242.24

White solid; $92 \%$ Yield (Procedure C); m.p. 87-90 ${ }^{\circ} \mathrm{C} ;{ }^{1} \mathrm{H} \mathrm{RMN}\left(\mathrm{CDCl}_{3}, 500 \mathrm{MHz}, \mathrm{ppm}\right) \delta=$ 4.40-4.33 (m, 2H), 4.19 (dd, $J=8 \mathrm{~Hz}, 2.5 \mathrm{~Hz}, 1 \mathrm{H}), 3.74$ (dd, $J=13 \mathrm{~Hz}, 6 \mathrm{~Hz}, 1 \mathrm{H}), 3.60$ (dd, $J$ $=12.5 \mathrm{~Hz}, 2.5 \mathrm{~Hz}, 1 \mathrm{H}), 1.56(\mathrm{~s}, 9 \mathrm{H}) ;{ }^{13} \mathrm{C} \mathrm{RMN}\left(\mathrm{CDCl}_{3}, 125 \mathrm{MHz}, \mathrm{ppm}\right) \delta=151.6,149.4$, 84.9, 64.6, 53.9, 51.9, 28.1; IR $\left(v=\mathrm{cm}^{-1}\right): 3669,2977,2899,2098,1801,1398,1363,1287$, 1078, 1066, 1016, 776; MS (ESI): m/z $265.2\left[\mathrm{M}+\mathrm{Na}^{+}\right]$; HRMS (ESI): m/z calcd for $\mathrm{C}_{9} \mathrm{H}_{14} \mathrm{~N}_{4} \mathrm{O}_{4} \mathrm{Na}[\mathrm{M}+\mathrm{Na}]^{+}$265.09073, found 265.09152.

\section{8 - tert-butyl (1-azido-3-hydroxypropan-2-yl)carbamate (known compound) ${ }^{8}$}


<smiles>NCC(CO)NC(=O)OCc1ccc([N+](=O)[O-])cc1</smiles>

Colourless oil; 84 \% Yield; (Procedure D); ${ }^{1} \mathrm{H} \mathrm{RMN}\left(\mathrm{CDCl}_{3}, 500 \mathrm{MHz}, \mathrm{ppm}\right) \delta=5.06$ (brs, 1H), 3.76 (brs, 1H), 3.72 (dd, $J=4.5 \mathrm{~Hz}, 11 \mathrm{~Hz}, 1 \mathrm{H}), 3.65$ (dd, $J=5 \mathrm{~Hz}, 11 \mathrm{~Hz}, 1 \mathrm{H}), 3.57-$

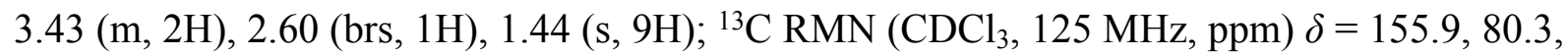
62.6, 51.7, 51.6, 28.5; IR $\left(v=\mathrm{cm}^{-1}\right): 3416,3346,2979,2930,2878,2102,1686,1516,1367$, 1285, 1251, 1166, 1060, 1026; MS (ESI): m/z 239.2 [M+Na+]; HRMS (ESI): m/z calcd for $\mathrm{C}_{8} \mathrm{H}_{16} \mathrm{~N}_{4} \mathrm{O}_{3} \mathrm{Na}[\mathrm{M}+\mathrm{Na}]^{+} 239.11146$, found 239.11258.

\title{
9 - tert-butyl 4-(((tert-butoxycarbonyl)amino)methyl)-2-oxooxazolidine-3-carboxylate
}

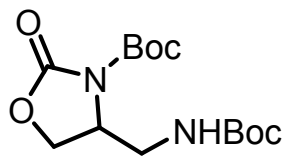

$$
\begin{gathered}
\mathrm{C}_{14} \mathrm{H}_{24} \mathrm{~N}_{2} \mathrm{O}_{6} \\
\text { Mol. Wt.: } 316.35
\end{gathered}
$$

White solid; $86 \%$ Yield (Procedure E); m.p. $150-152{ }^{\circ} \mathrm{C} ;{ }^{1} \mathrm{H} \mathrm{RMN}\left(\mathrm{CDCl}_{3}, 500 \mathrm{MHz}, \mathrm{ppm}\right) \delta$ $=4.85($ brs, $1 \mathrm{H}), 4.37-4.31(\mathrm{~m}, 2 \mathrm{H}), 4.27(\mathrm{dd}, J=8 \mathrm{~Hz}, 2 \mathrm{~Hz}, 1 \mathrm{H}), 3.54-3.45(\mathrm{~m}, 2 \mathrm{H}), 1.56(\mathrm{~s}$, 9H), $1.44(\mathrm{~s}, 9 \mathrm{H}) ;{ }^{13} \mathrm{C} \mathrm{RMN}\left(\mathrm{CDCl}_{3}, 125 \mathrm{MHz}, \mathrm{ppm}\right) \delta=156.5,152.2,149.6,84.5,80.4$, 65.0, 55.3, 42.1, 28.4, 28.1; IR $\left(v=\mathrm{cm}^{-1}\right): 3370,2983,2963,2933,1821,1699,1522,1388$, 1304, 1163, 1088, 1055, 766; MS (ESI): m/z 339.3 [M+Na ${ }^{+}$; HRMS (ESI): m/z calcd for $\mathrm{C}_{14} \mathrm{H}_{24} \mathrm{~N}_{2} \mathrm{O}_{6} \mathrm{Na}[\mathrm{M}+\mathrm{Na}]^{+}$339.15266, found 339.15384.

\section{0 - Ethyl 1-((2-oxooxazolidin-4-yl)methyl)-1H-1,2,3-triazole-4-carboxylate}

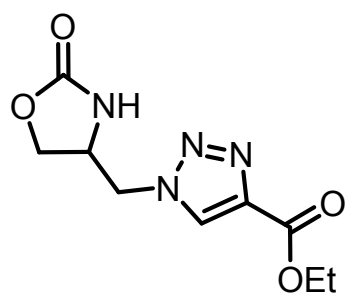

\author{
$\mathrm{C}_{9} \mathrm{H}_{12} \mathrm{~N}_{4} \mathrm{O}_{4}$
}

Mol. Wt.: 240.22

Pink white solid; 83 \% Yield (Procedure F); m.p. 146-148 ${ }^{\circ} \mathrm{C} ;{ }^{1} \mathrm{H}$ RMN (MeOD, $500 \mathrm{MHz}$, ppm) $\delta=8.56(\mathrm{~s}, 1 \mathrm{H}), 4.68-4.60(\mathrm{~m}, 2 \mathrm{H}), 4.55(\mathrm{t}, J=8.5 \mathrm{~Hz}, 1 \mathrm{H}), 4.46-4.41(\mathrm{~m}, 1 \mathrm{H}), 4.39(\mathrm{q}$, $J=7 \mathrm{~Hz}, 2 \mathrm{H}), 4.33$ (dd, $J=4.5 \mathrm{~Hz}, 9 \mathrm{~Hz}, 1 \mathrm{H}), 1.38$ (t, $J=7 \mathrm{~Hz}, 3 \mathrm{H}) ;{ }^{13} \mathrm{C} \mathrm{RMN}(\mathrm{MeOD}, 125$ $\mathrm{MHz}, \mathrm{ppm}) \delta=161.9,161.5,141.0,130.6,68.6,62.3,54.2,53.3,14.5$; IR $\left(v=\mathrm{cm}^{-1}\right): 3316$, 3138, 2984, 2928, 2853, 1727, 1216, 1044; MS (ESI): m/z 239.3 [M-H']; HRMS (ESI): m/z calcd for $\mathrm{C}_{9} \mathrm{H}_{11} \mathrm{~N}_{4} \mathrm{O}_{4}\left[\mathrm{M}-\mathrm{H}^{+}\right]$239.07748, found 239.07811. 
IV. ${ }^{1} \mathrm{H}$ and ${ }^{13} \mathrm{C}$ NMR spectra

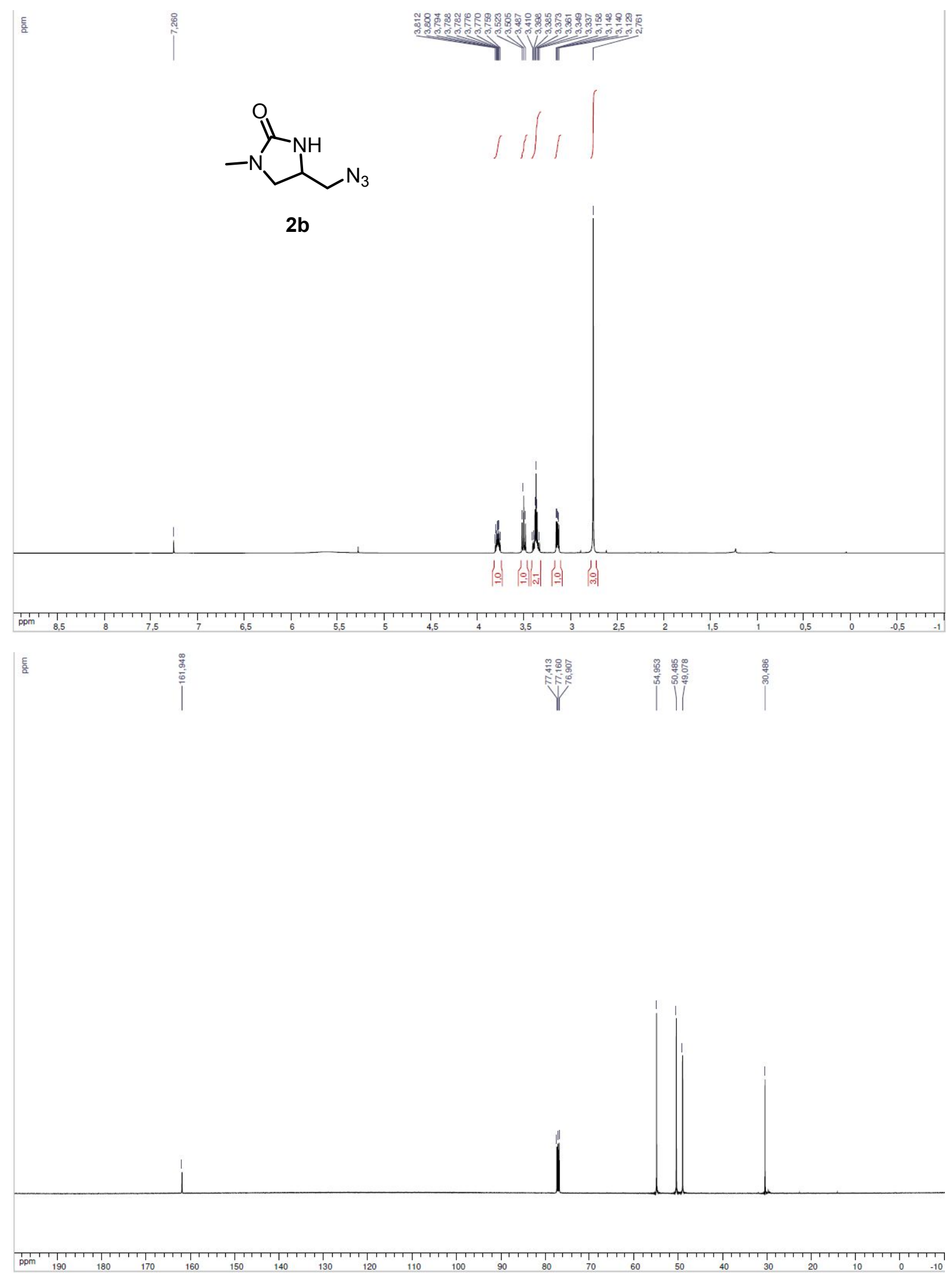




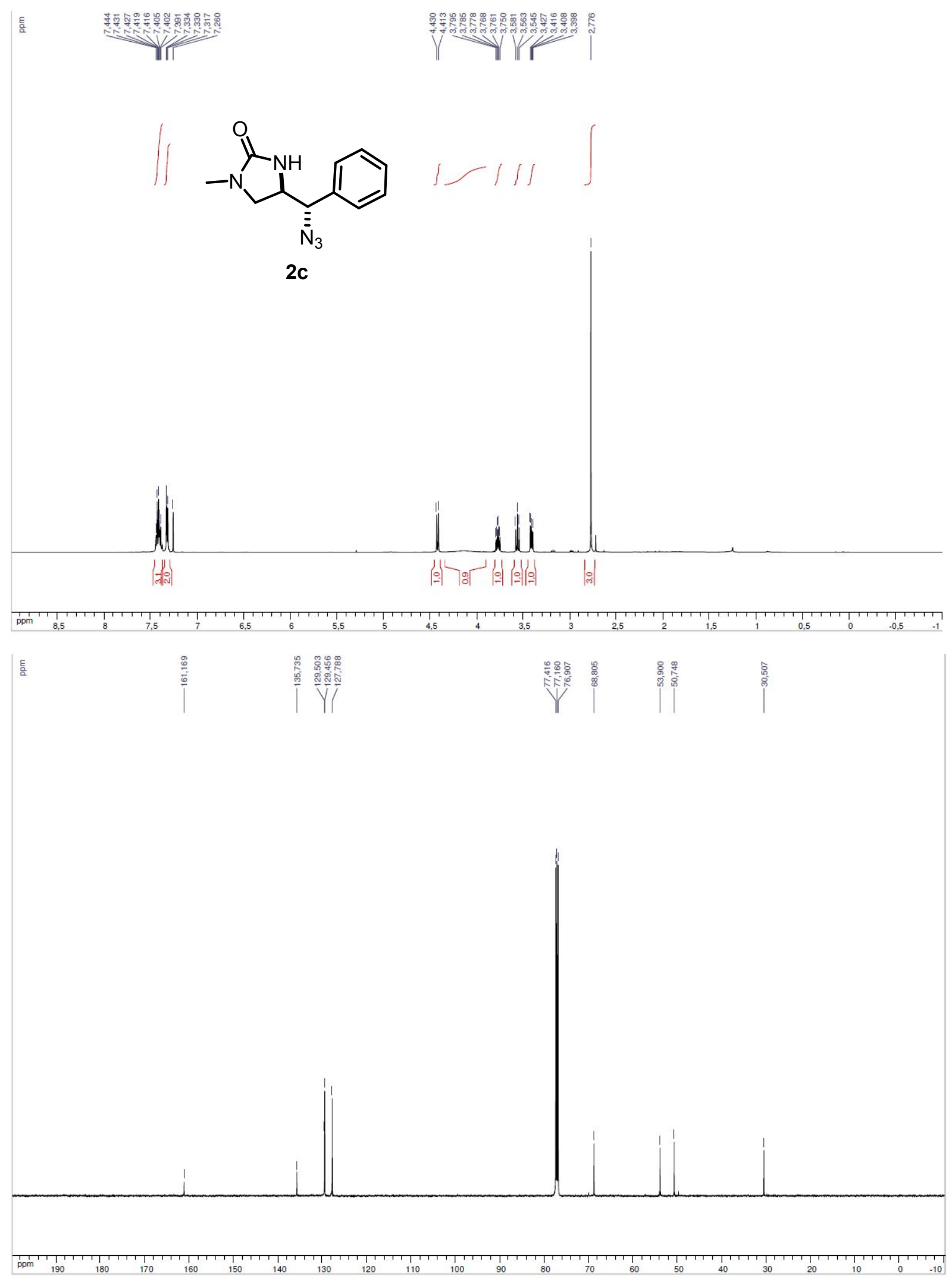




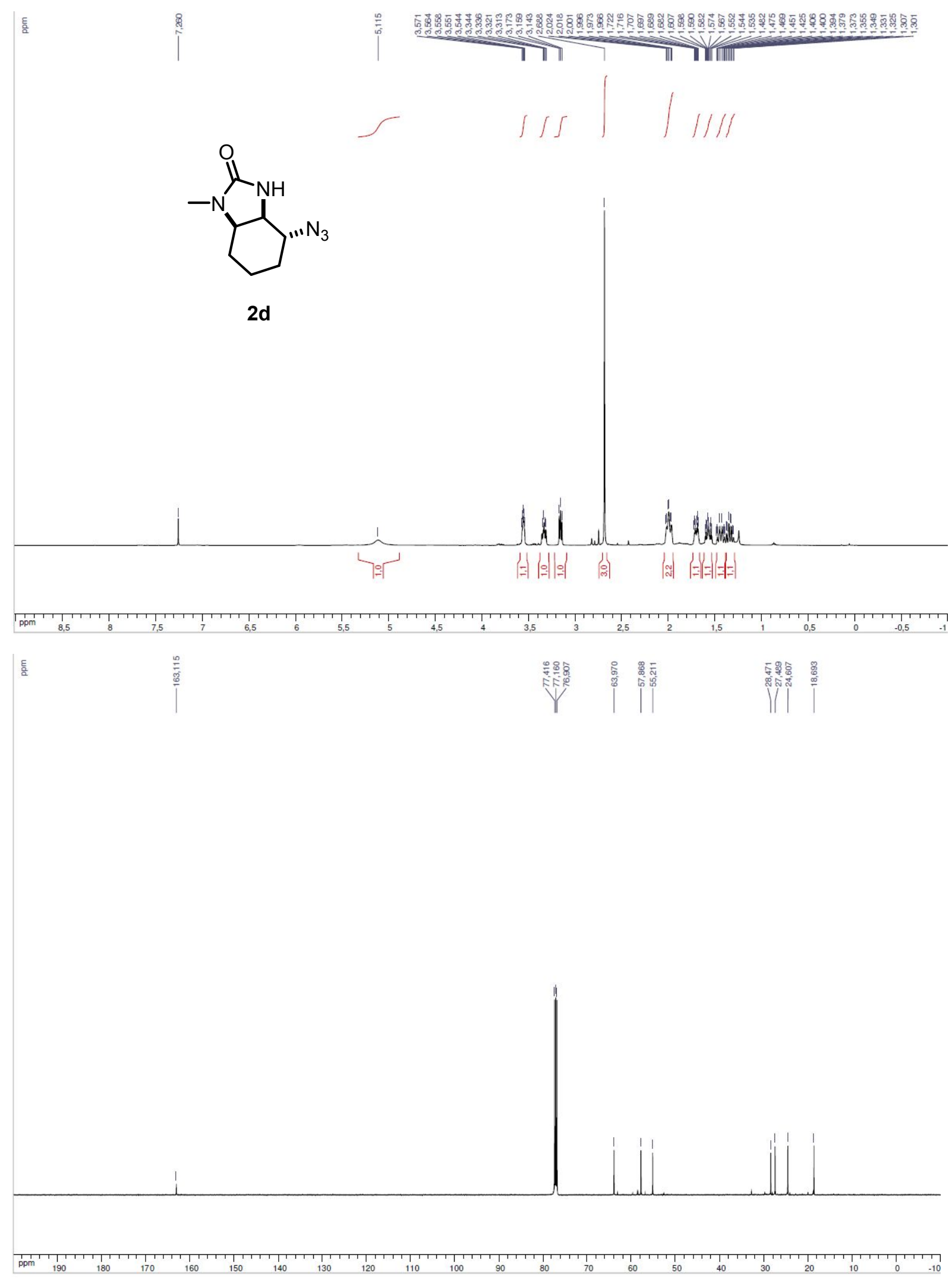




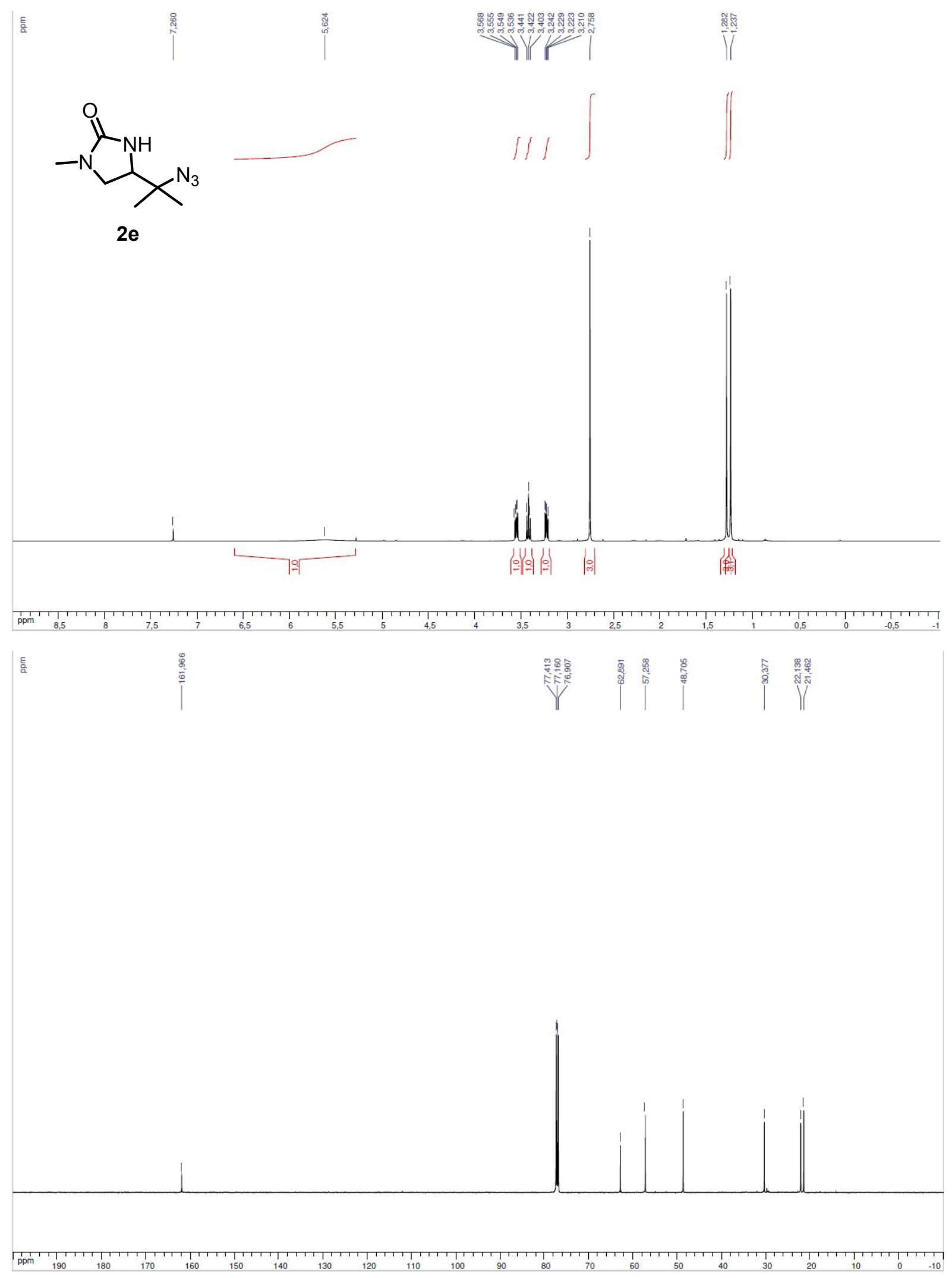




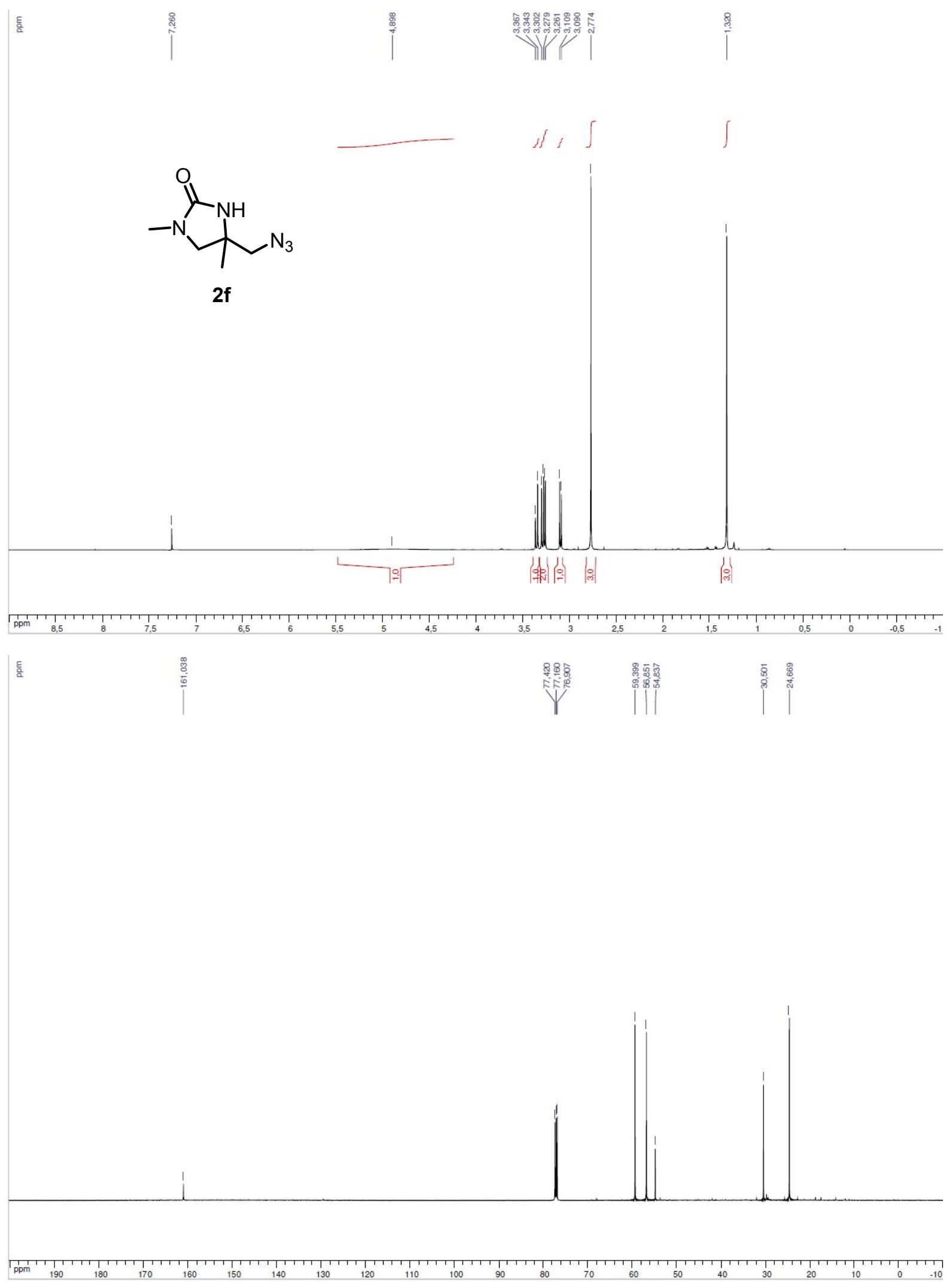

S27 


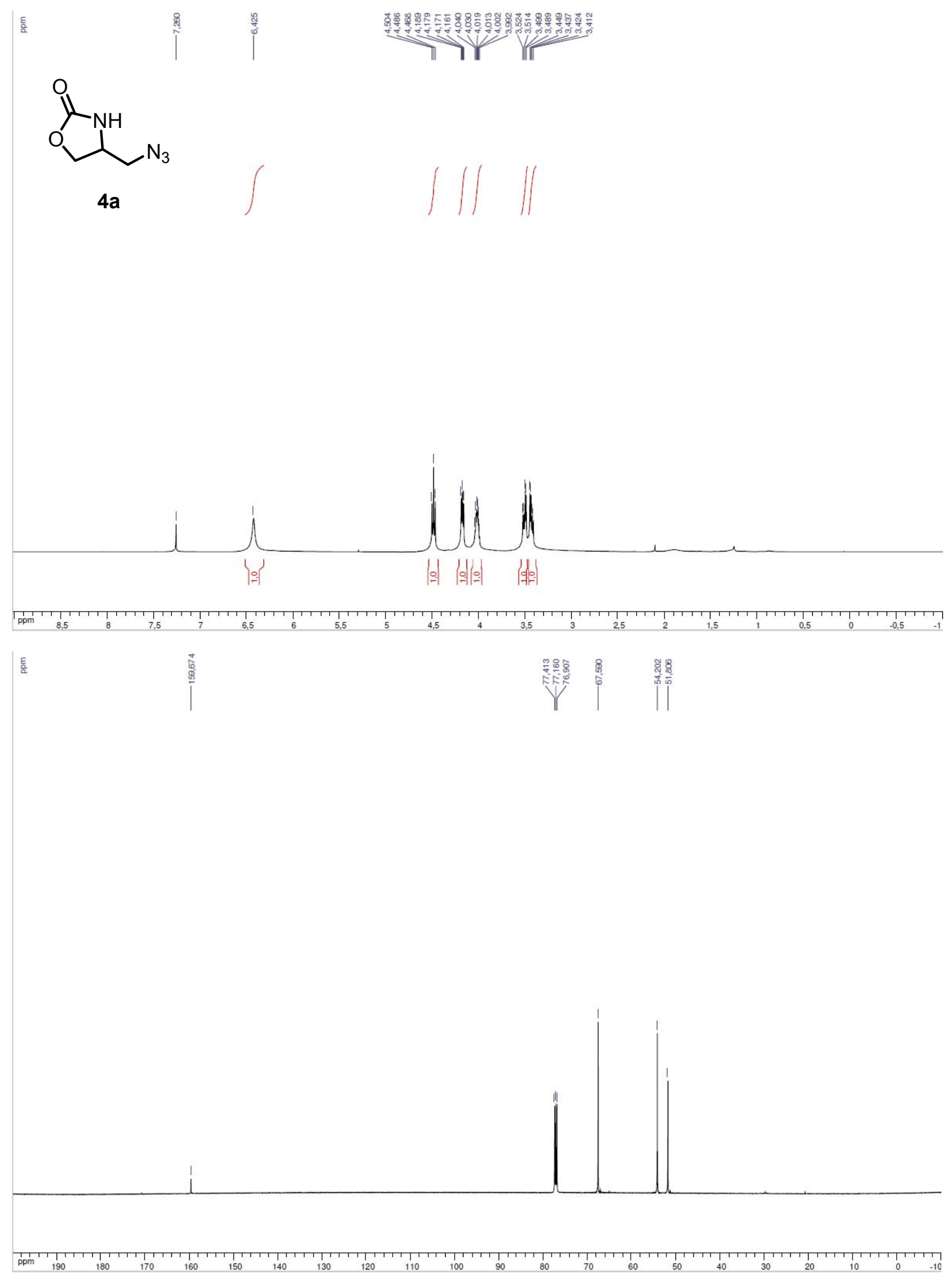



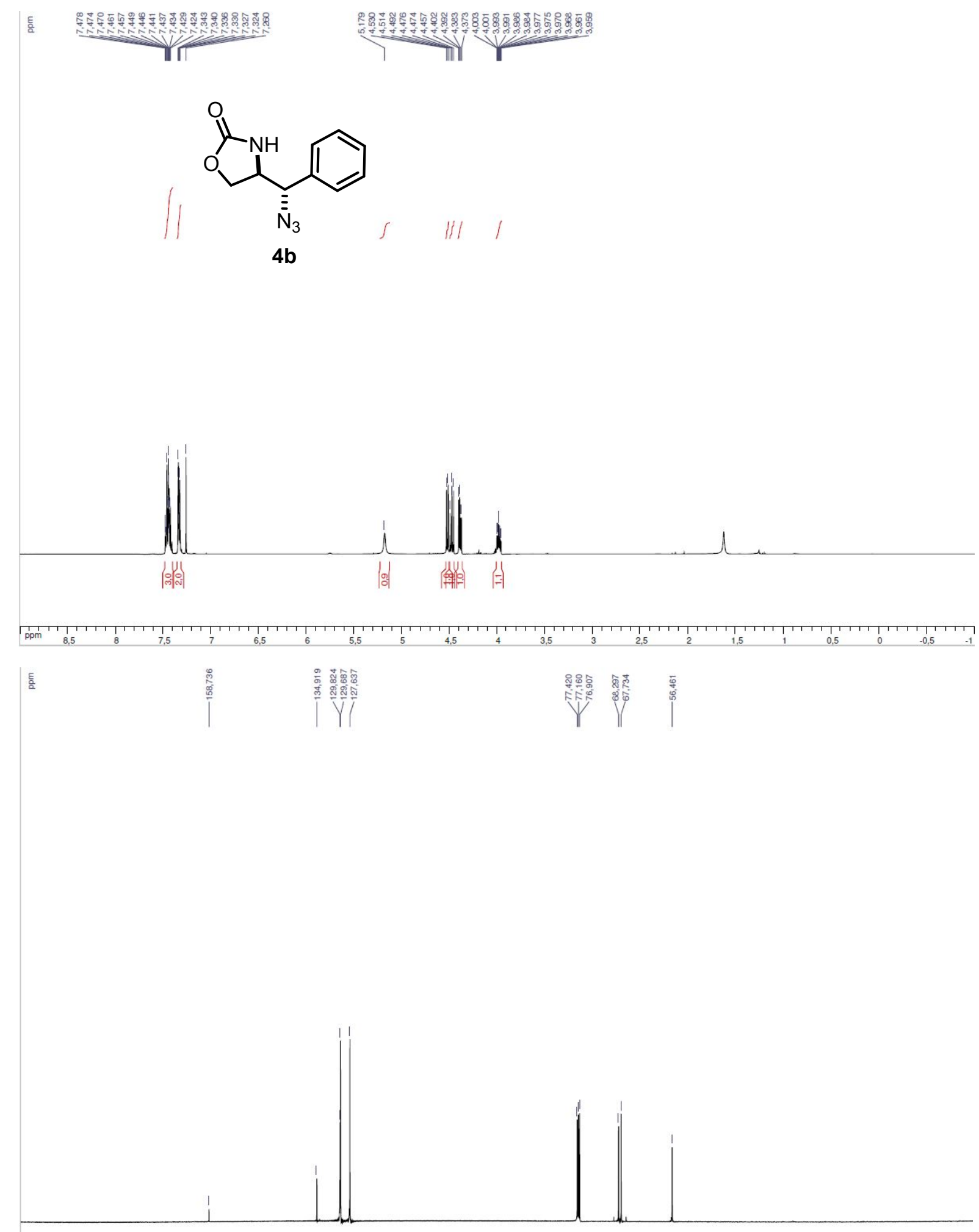

S29 


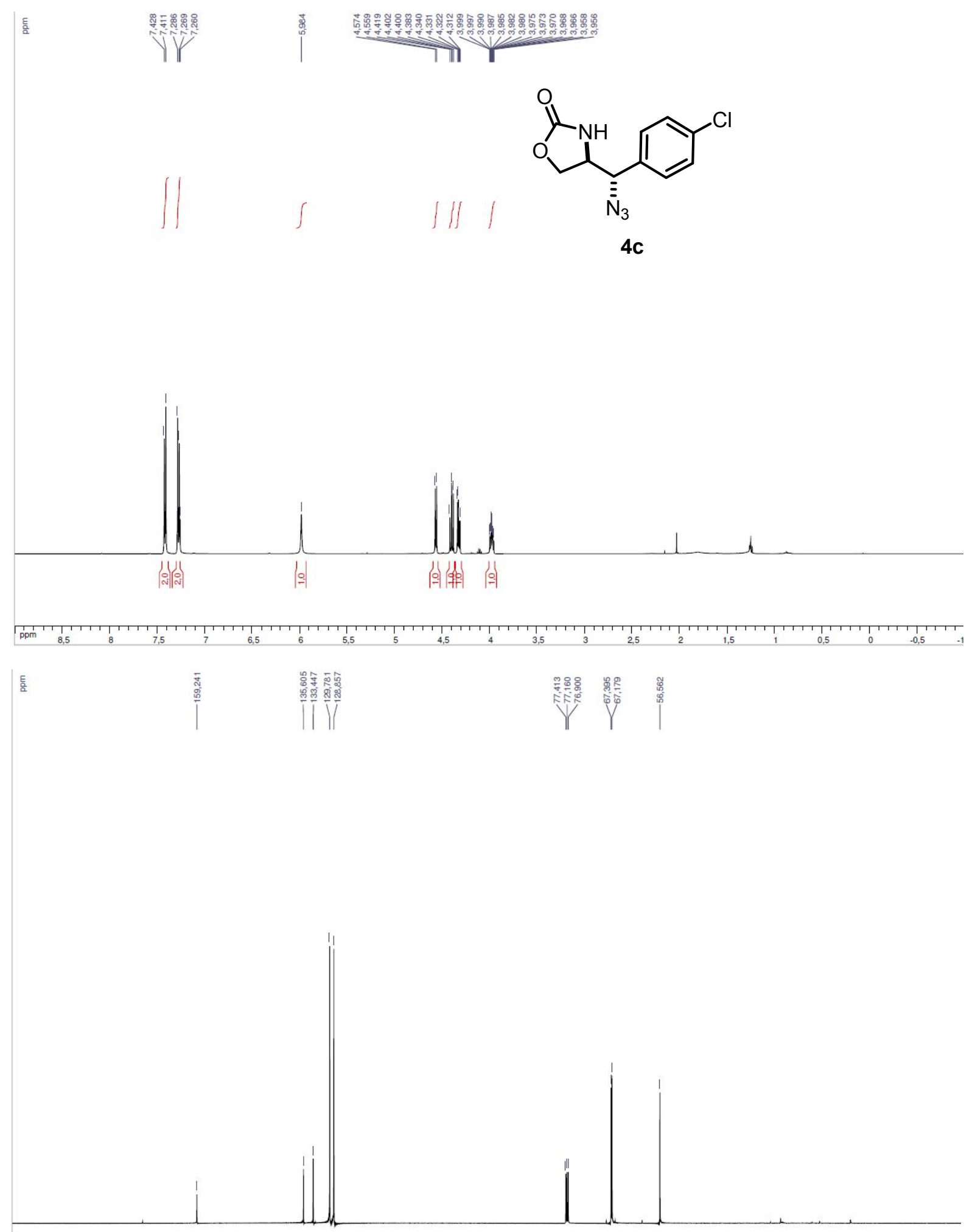

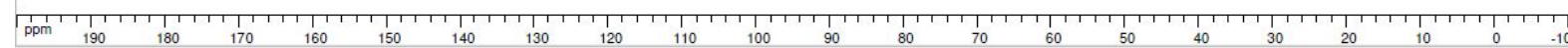




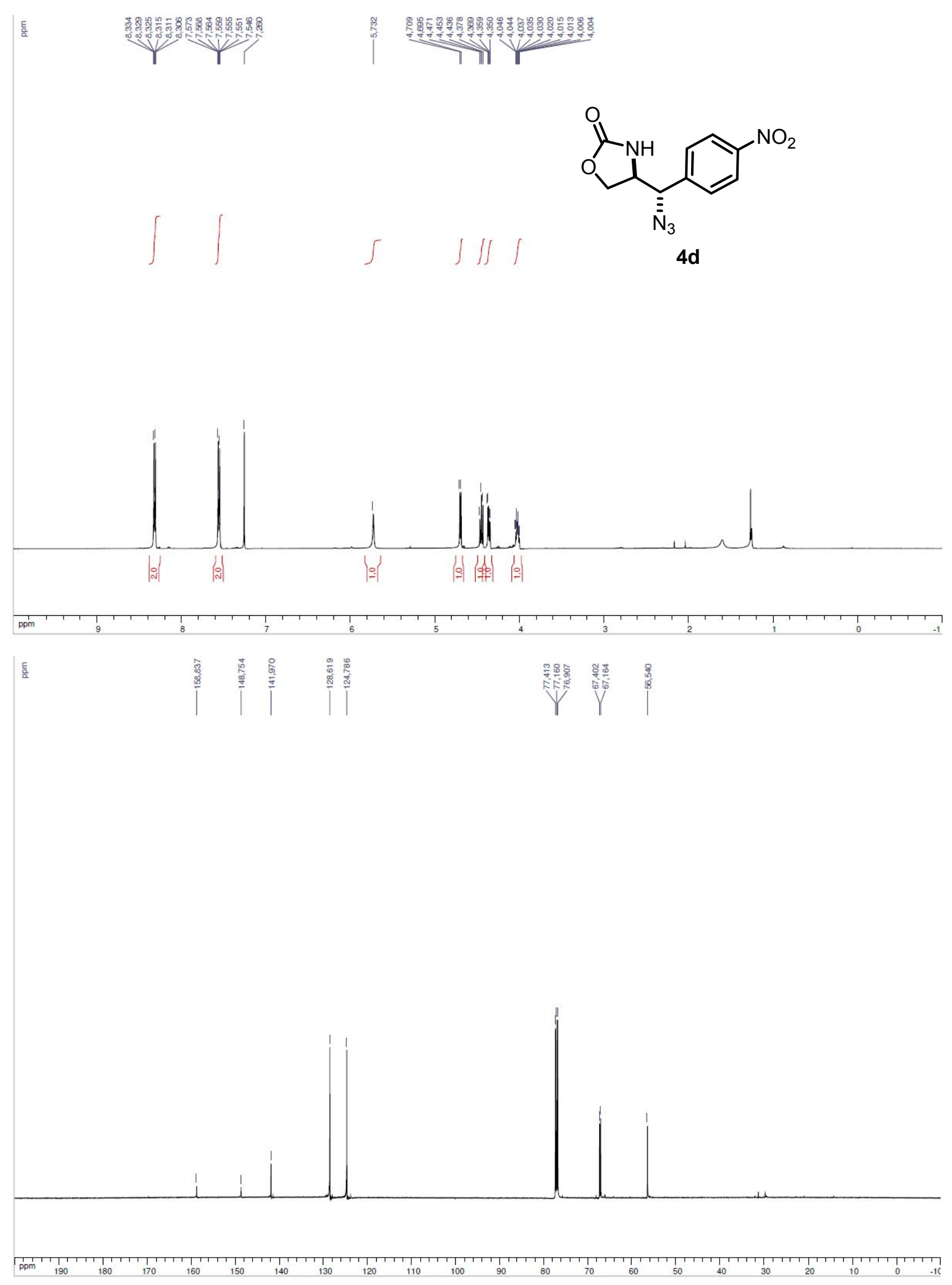




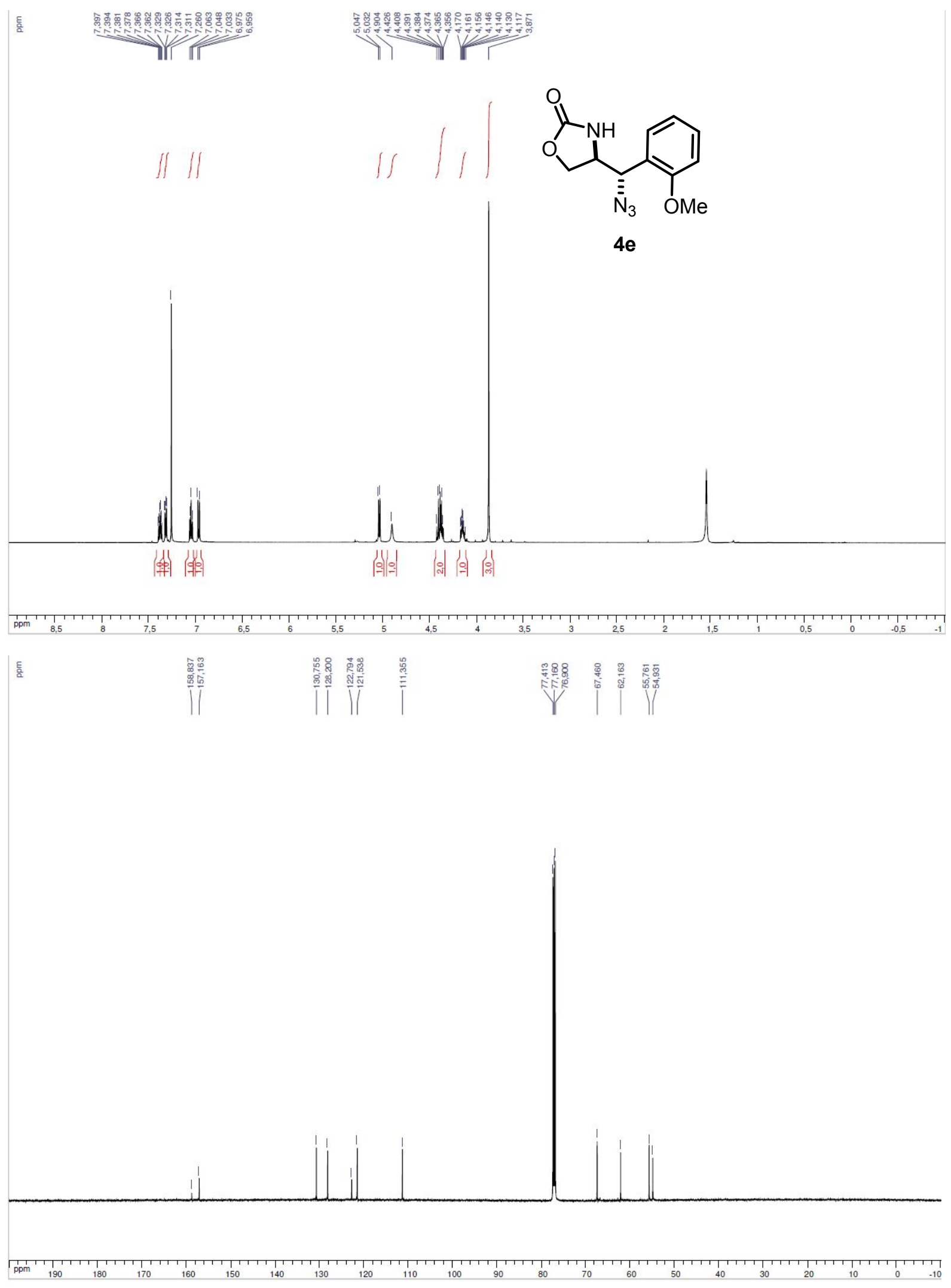

S32 


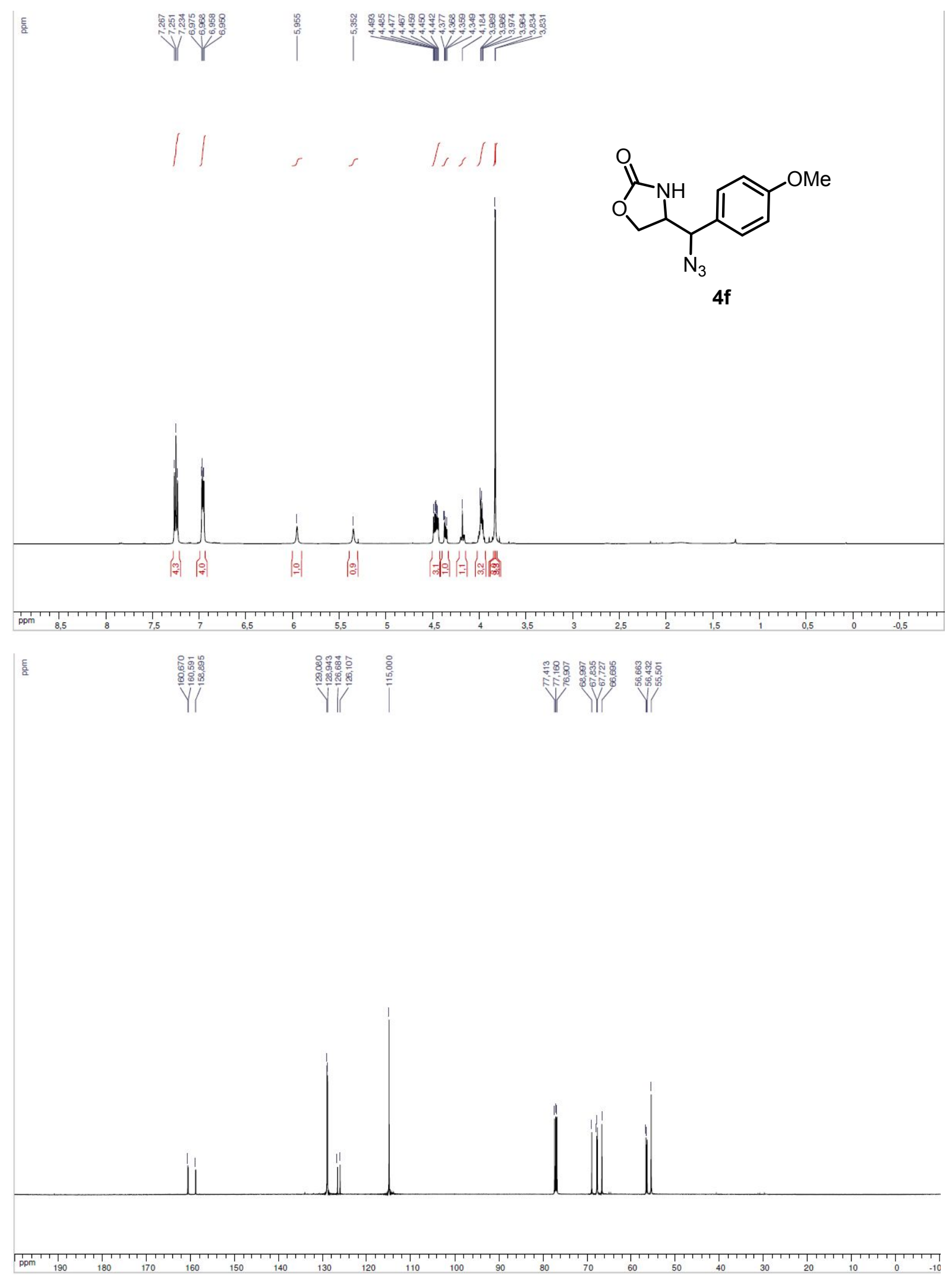




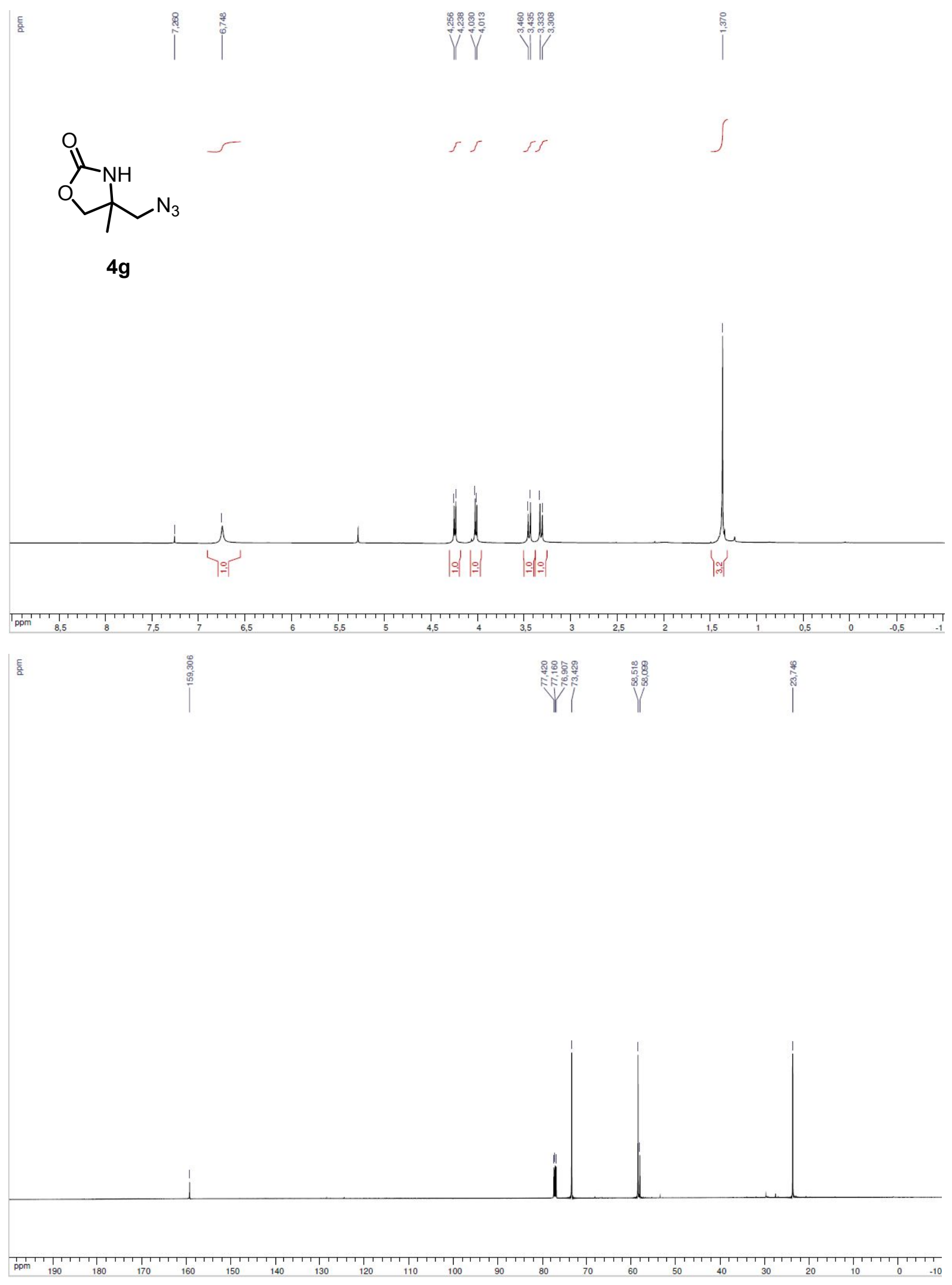

S34 


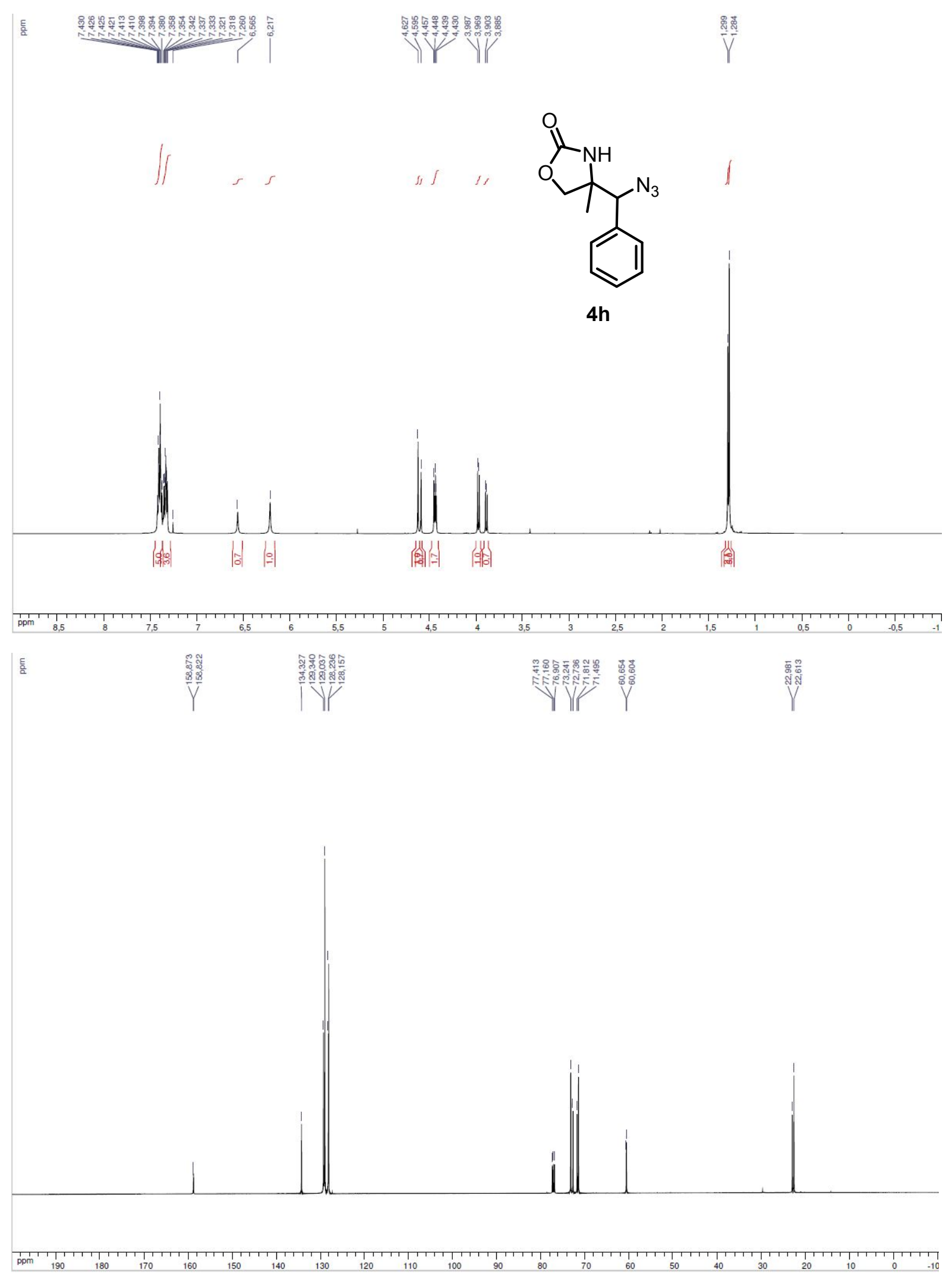




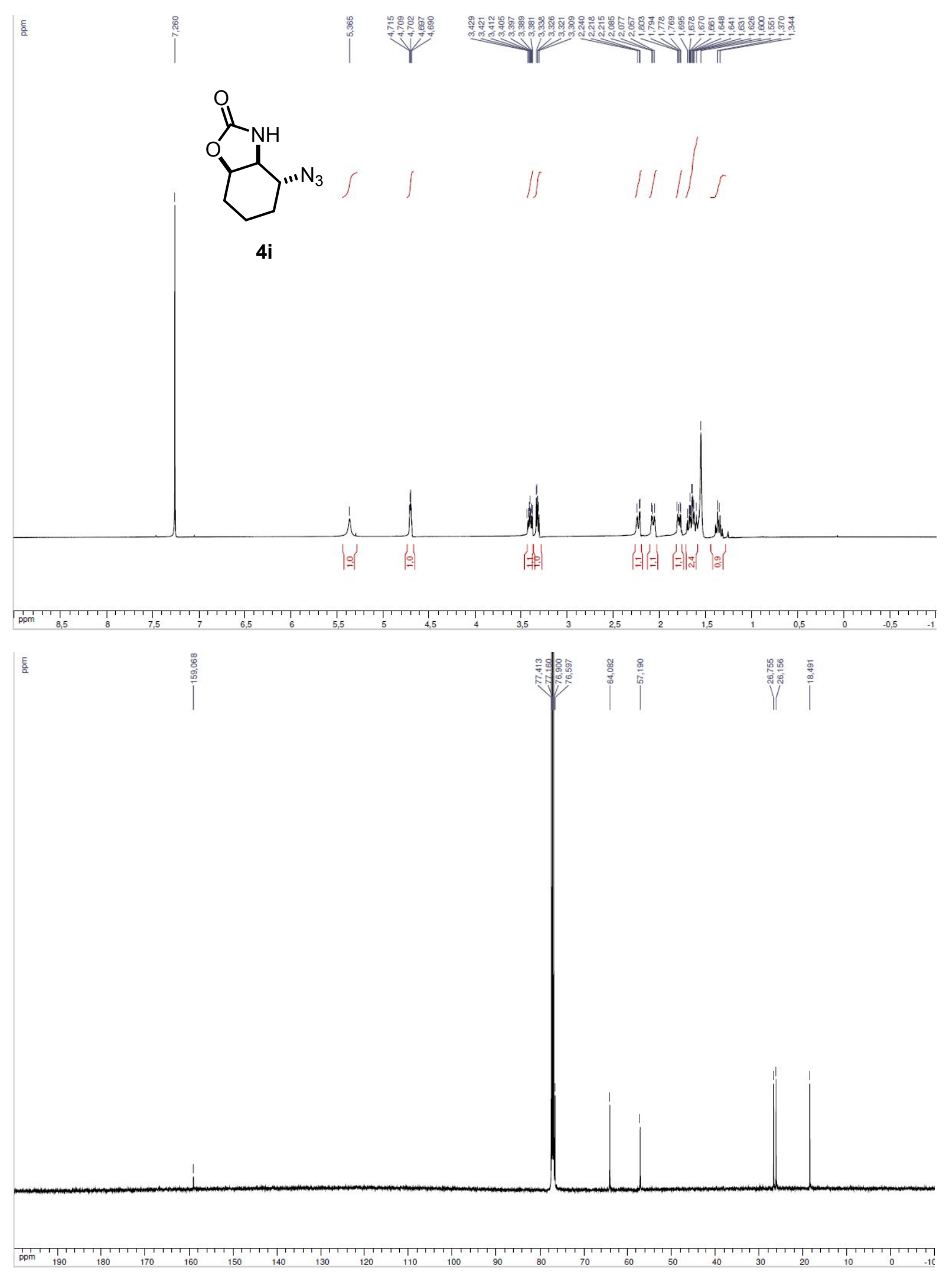




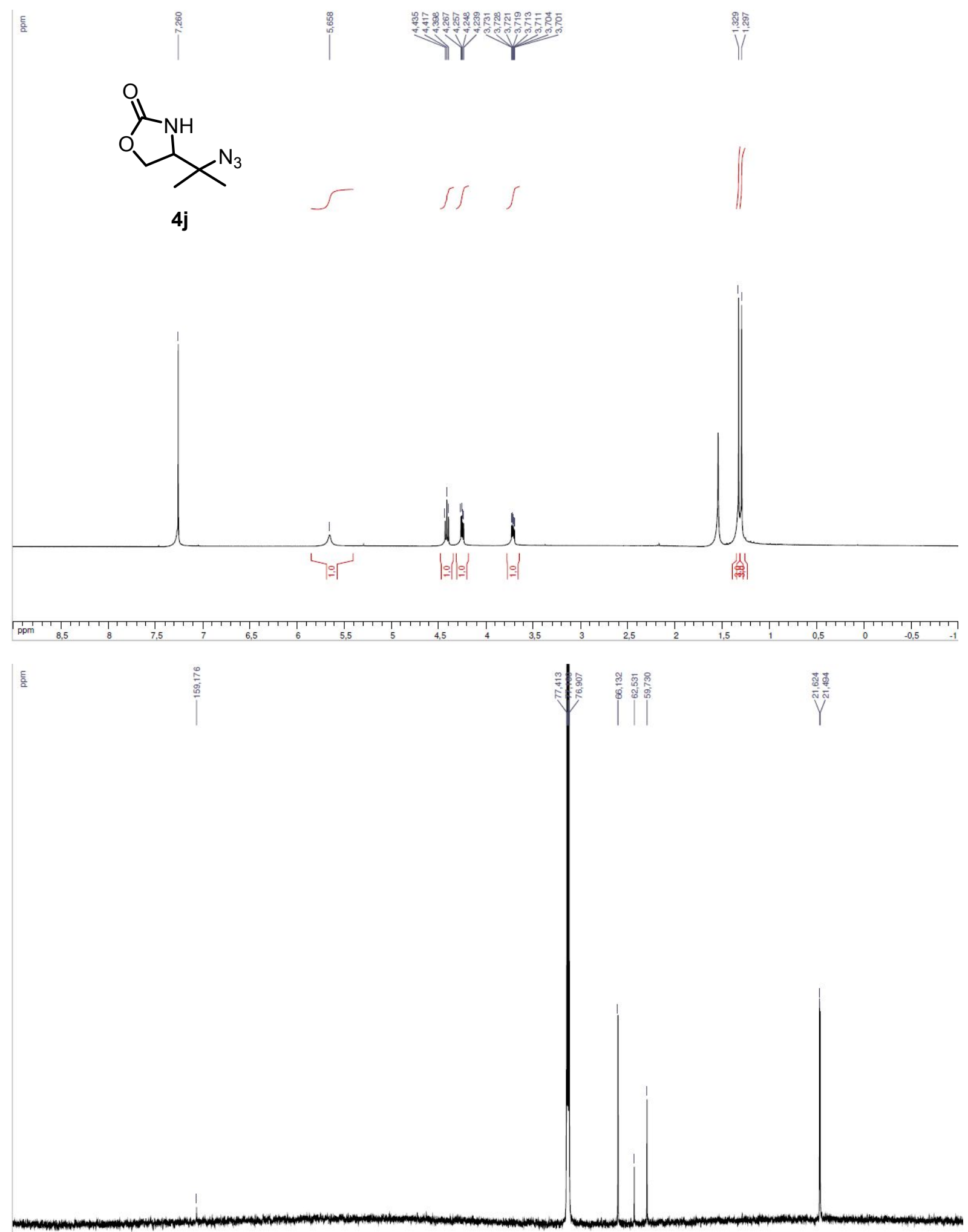

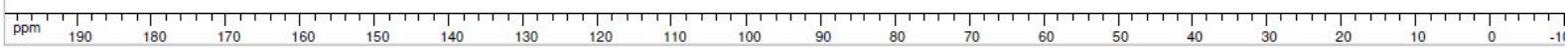




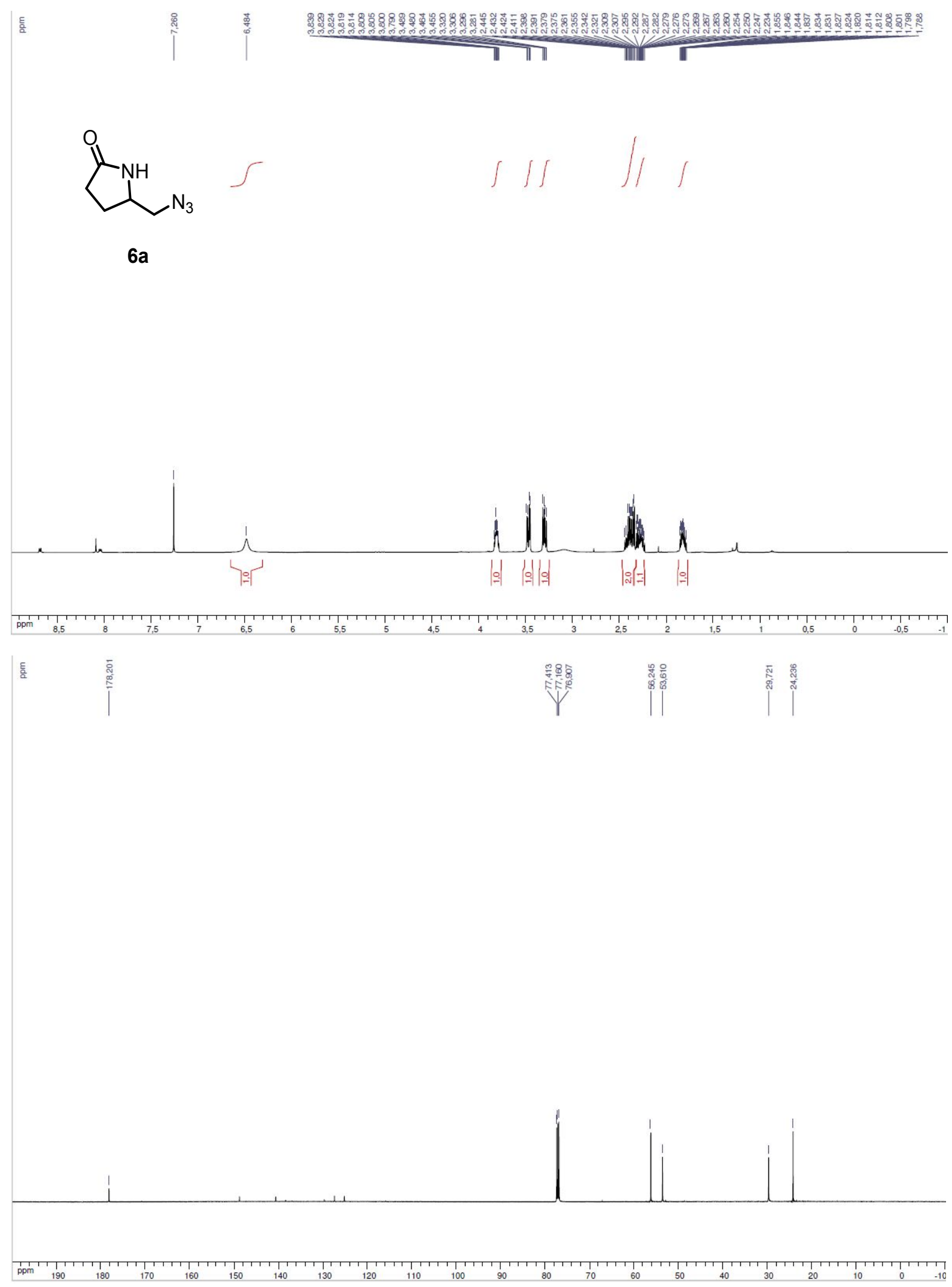

S38 


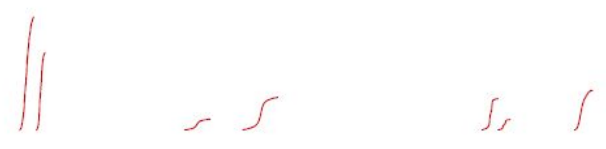<smiles>N[C@@H](c1ccccc1)C1CCC(=O)N1</smiles>

6b
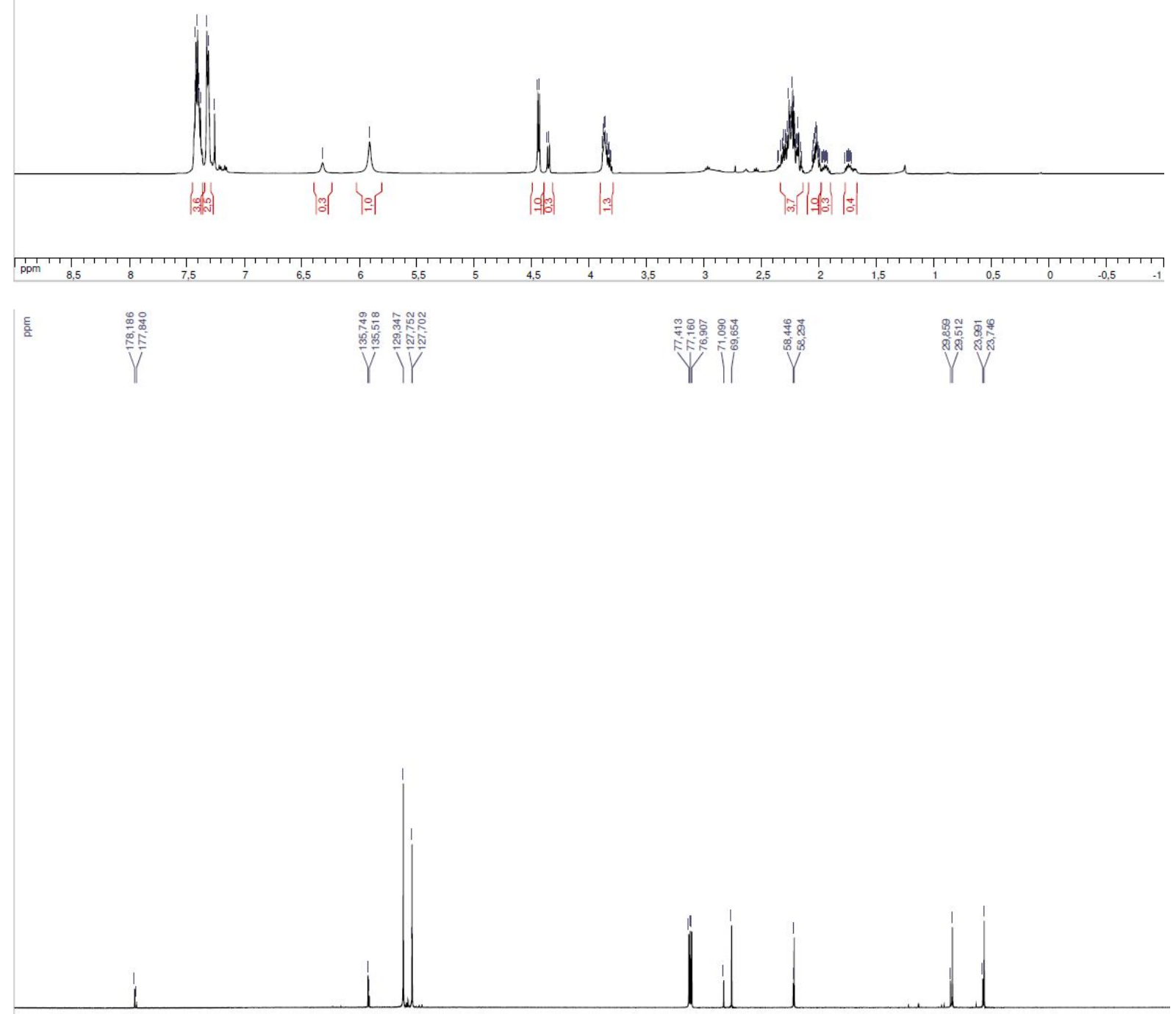

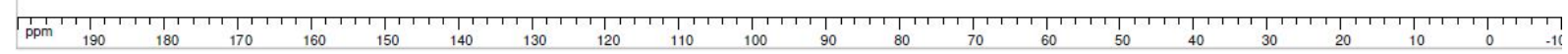




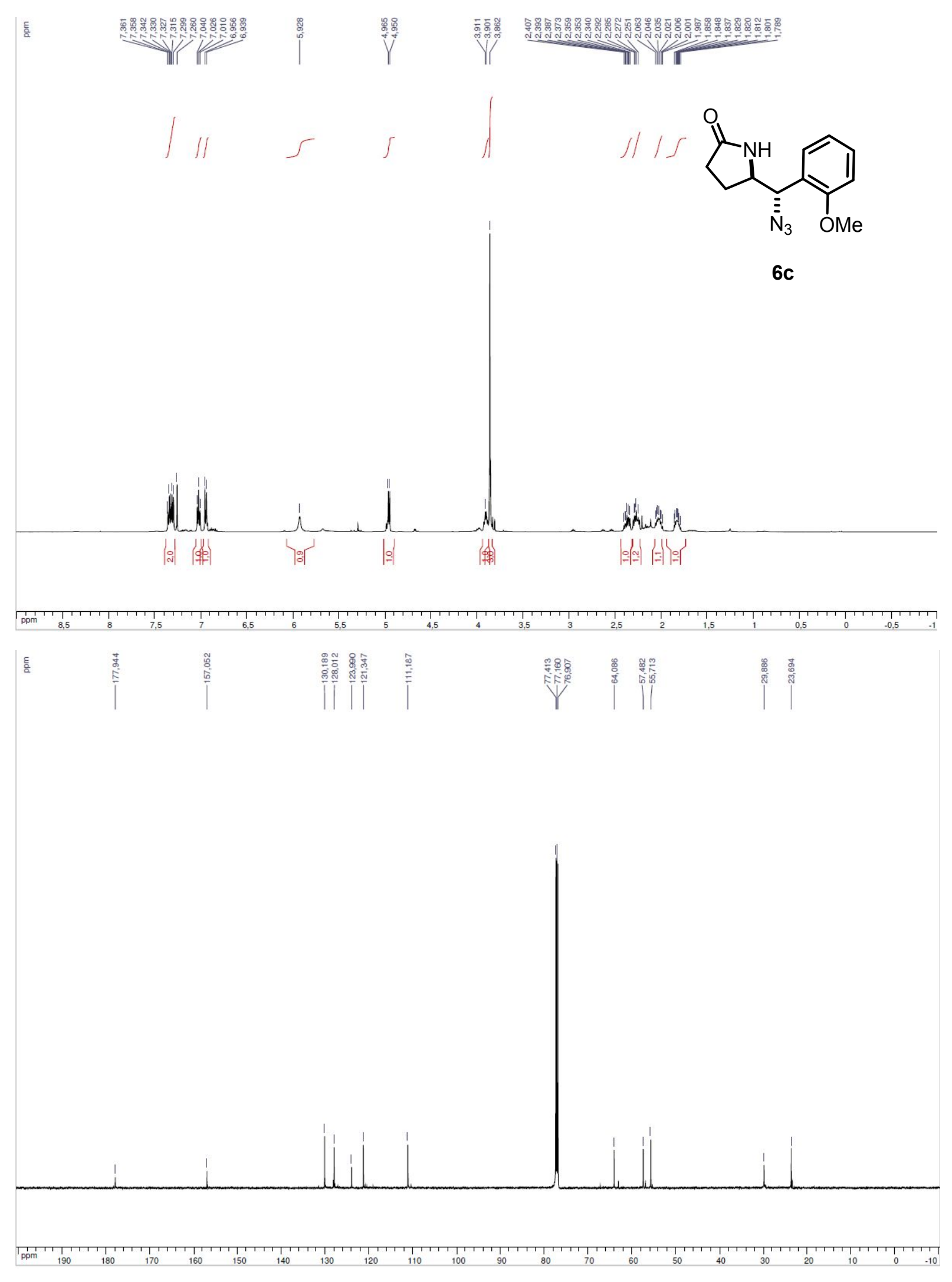




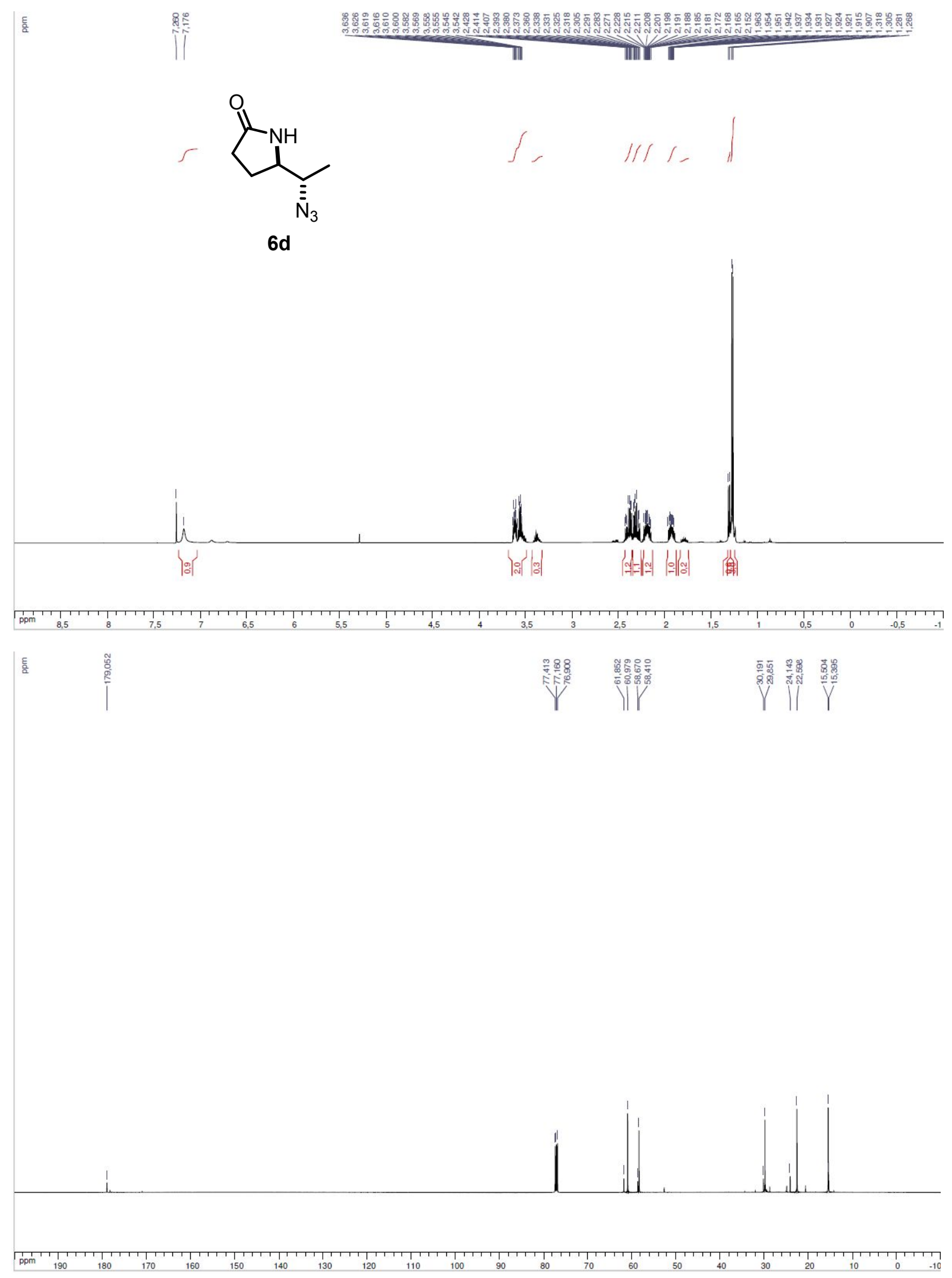




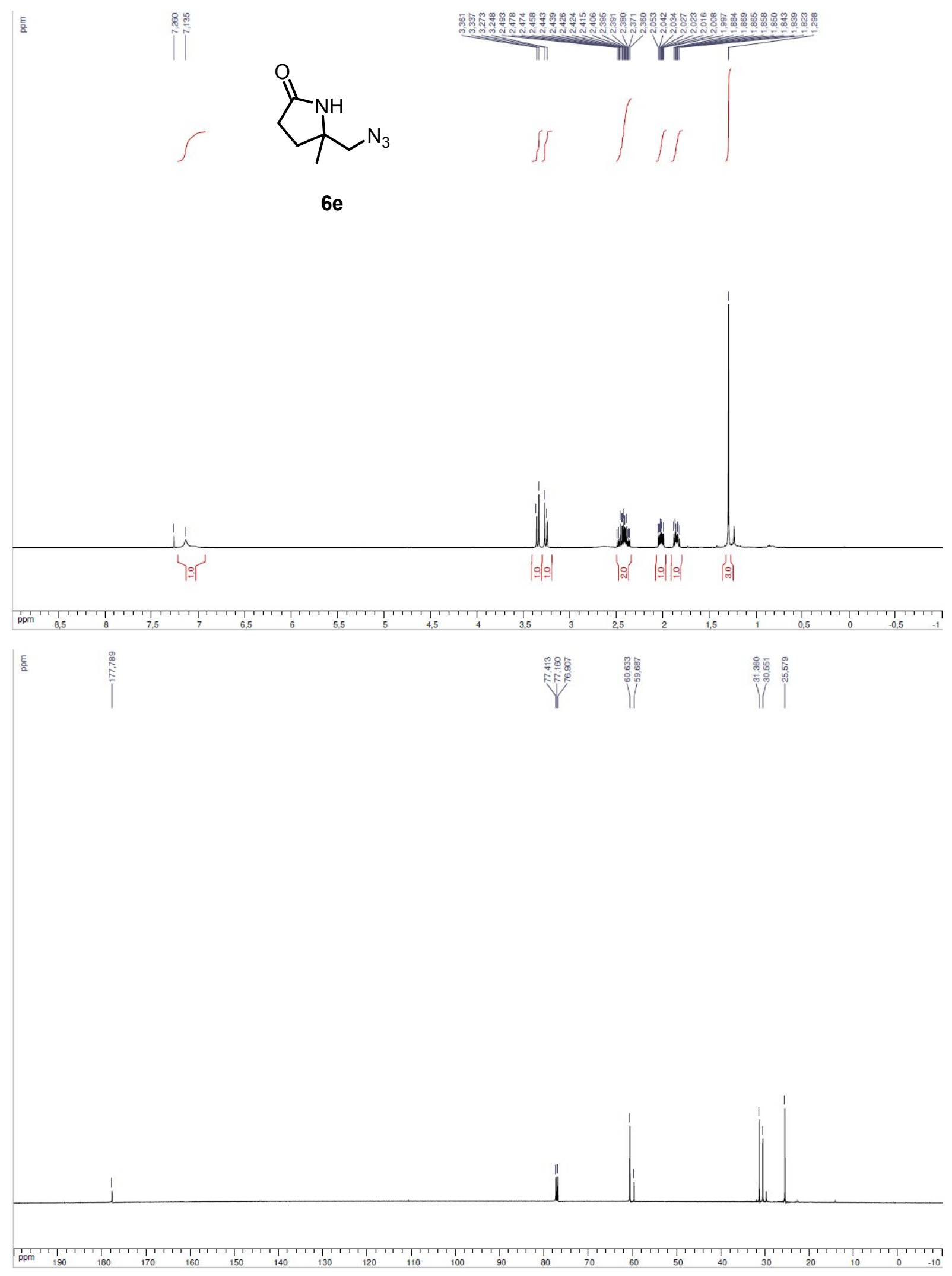




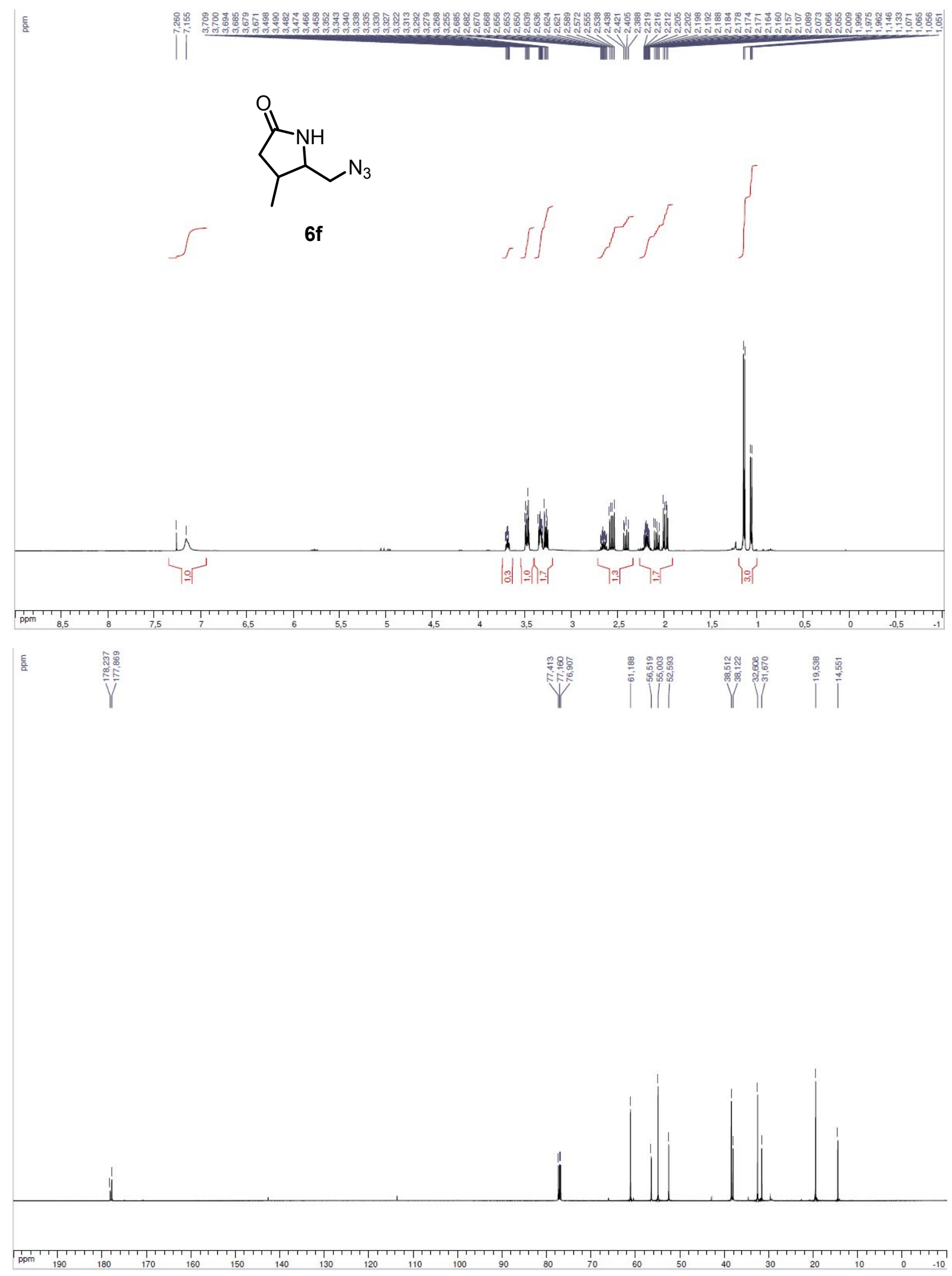




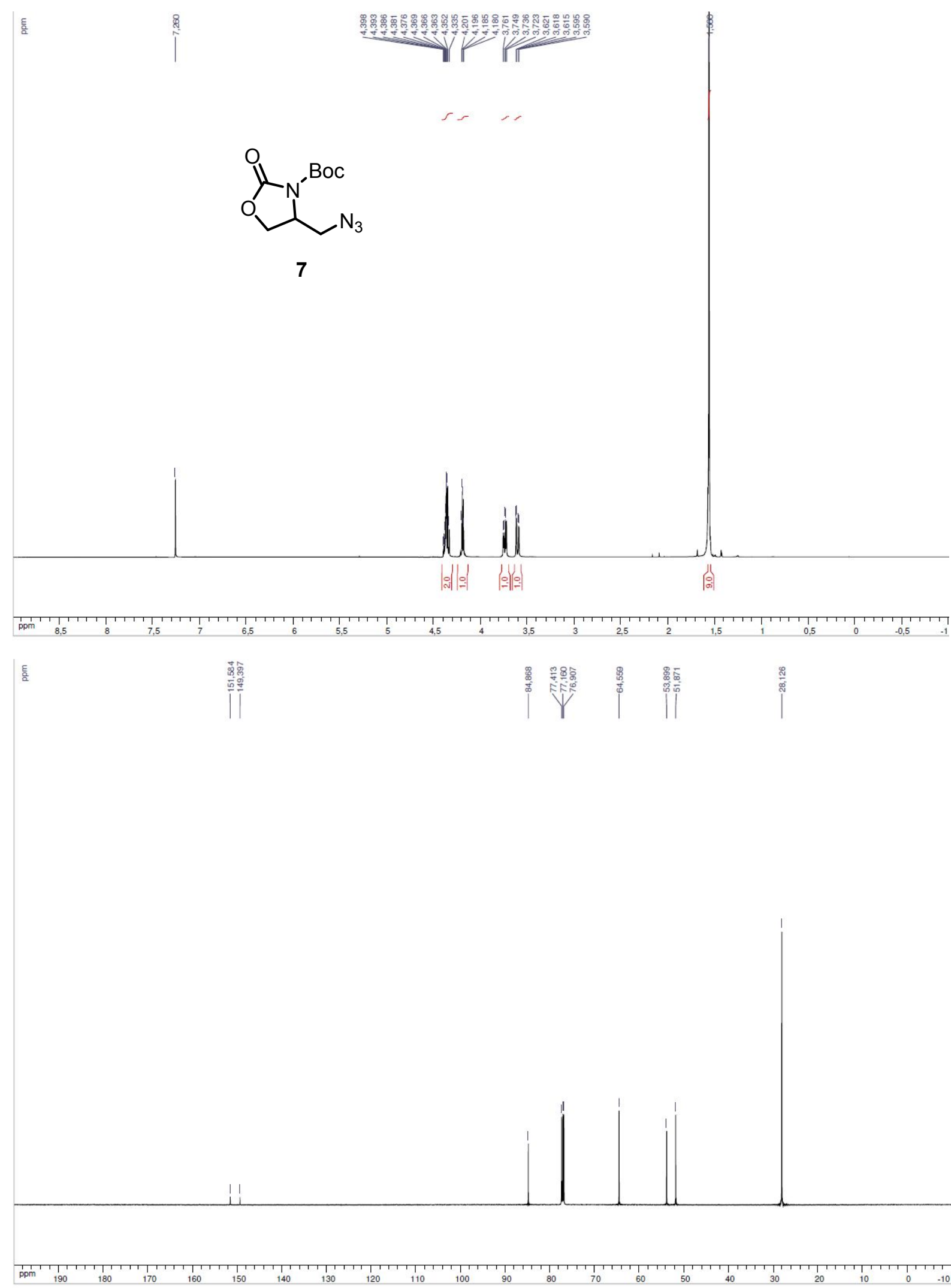




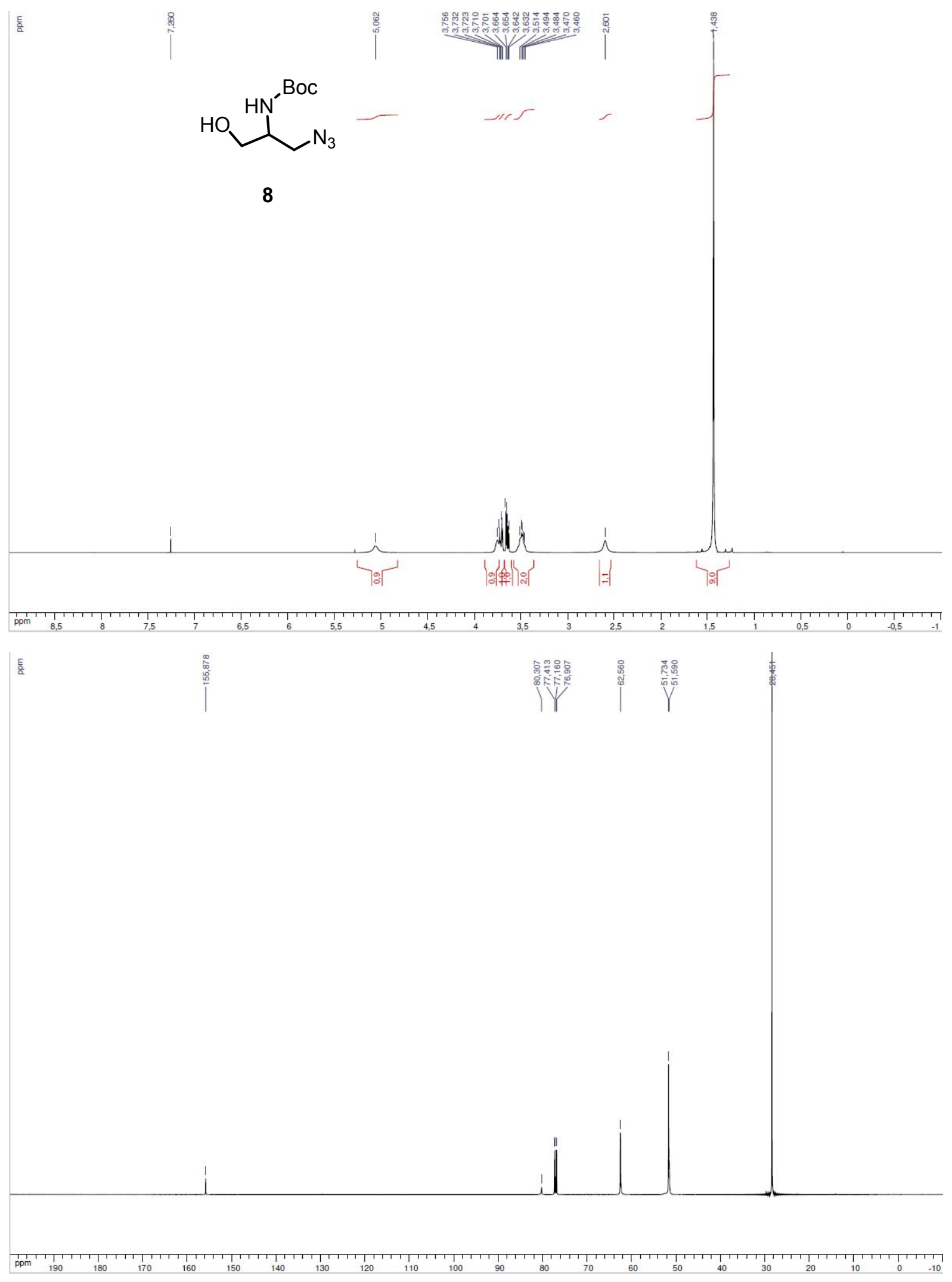

S45 


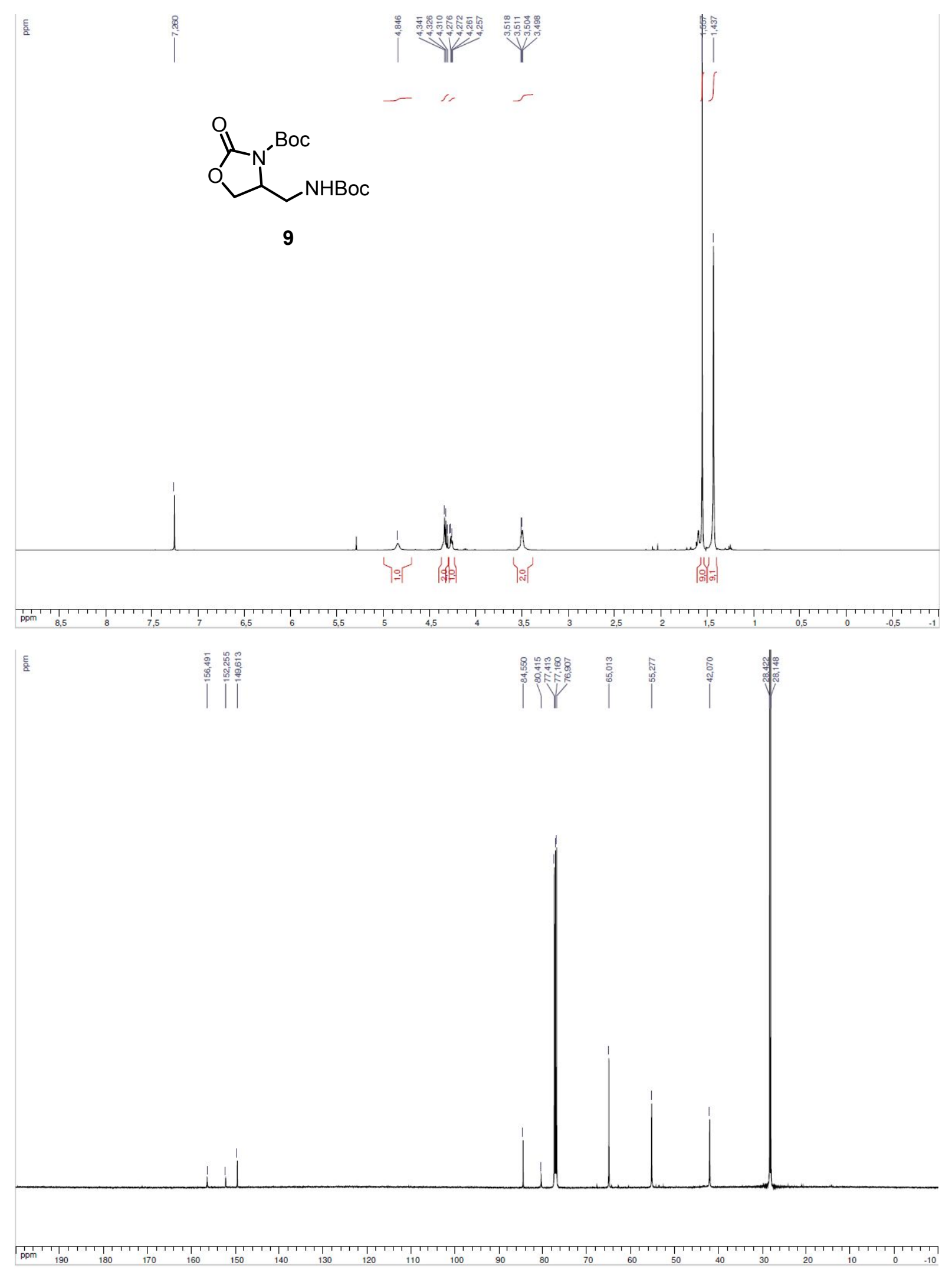




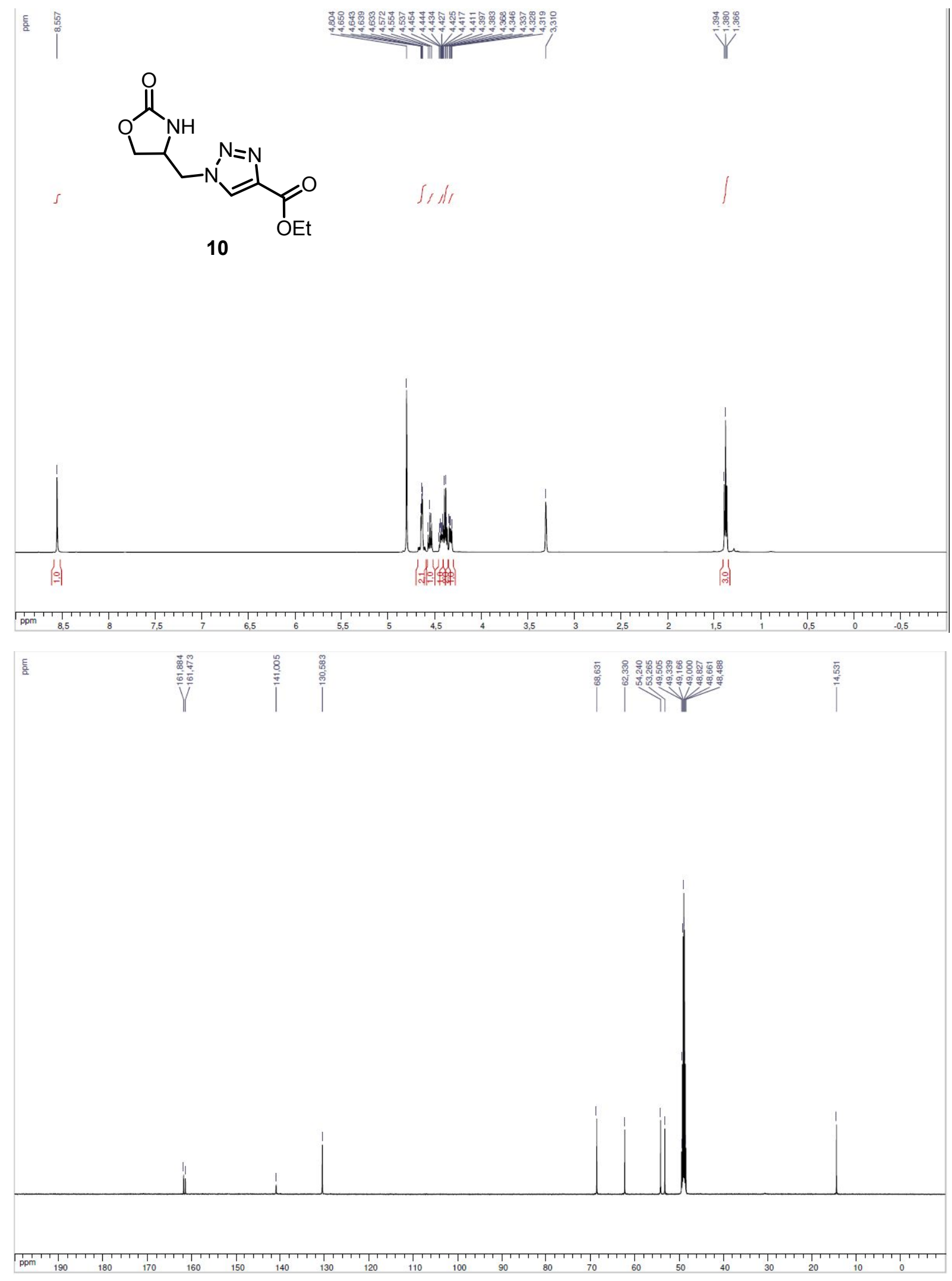




\section{References}

${ }^{1}$ Donohoe, T. J.; Callens, C. K. A.; Thompson, A. L. Tethered aminohydroxylation (TA) reaction of amides Org. Lett. 2009, 11, 2305-2307.

${ }^{2}$ Lo, K.-Y.; Ye, L.; Yang, D. Palladium-catalyzed aerobic oxidative cyclization of aliphatic alkenyl amides for the construction of pyrrolizidine and indolizidine derivatives Synlett, 2017, 28, 1570-1575.

${ }^{3}$ Genzyme Coporation: Siegel, C.; Gimi, R.; Reardon, M.; Zhao, J. Method of preparing glucosylceramide synthase inhibitors WO2014/151291, 2014, A1.

${ }^{4}$ Manick, A.-D.; Aubert, S.; Yalcouye, B.; Prangé, T.; Berhal, F.; Prestat, G. Access to functionalized imidazolidin-2-one derivatives by iron-catalyzed oxyamination of alkenes Chem. Eur. J. 2018, 24, 11485-11492.

${ }^{5}$ Jiang, H.; Lang, K.; Lu, H.; Wojtas, L.; Zhang, X. P. Asymmetric radical bicyclization of allyl azidoformates via cobalt(II)-based metalloradical catalysis J. Am. Chem. Soc. 2017, 139, 9164-9167.

${ }^{6}$ Liu, R.; Herron, S. R.; Fleming, S; A. Copper-catalyzed tethered aziridination of unsaturated $N$-Tosyloxy carbamates J. Org. Chem. 2007, 72, 5587-5591.

${ }^{7}$ Brand, J. P.; Siles, J. I. O.; Waser, J. Synthesis of chiral bifunctional (thio)urea $N$-heterocyclic carbenes Synlett, 2010, 881-884.

${ }^{8}$ Jain, D. R.; Ganesh, K. N. Clickable $C^{\gamma}$-azido(methylene/butylene) peptide nucleic acids and their clicked fluorescent derivatives: synthesis, DNA hybridization properties, and cell penetration studies J. Org. Chem. 2014, 79, 6708-6714. 